\title{
PROPERTIES OF INTERPLANETARY CORONAL MASS EJECTIONS AT ONE AU DURING 1995-2004
}

\author{
L. JIAN \\ Institute of Geophysics and Planetary Physics, University of California, Los Angeles, \\ 595 Charles E. Young Dr. East, 6862 Slichter, Los Angeles, CA 90095, U.S.A. \\ (e-mail: jlan@igpp.ucla.edu) \\ C.T. RUSSELL \\ Institute of Geophysics and Planetary Physics, University of California, Los Angeles, \\ 595 Charles E. Young Dr. East, 6869 Slichter, Los Angeles, CA 90095, U.S.A. \\ (e-mail: ctrussel@igpp.ucla.edu) \\ J.G. LUHMANN \\ Space Sciences Laboratory, University of California, Berkeley, CA 94720, U.S.A. \\ (e-mail: jgluhman@ssl.berkeley.edu) \\ and \\ R.M. SKOUG \\ Los Alamos National Laboratory, Los Alamos, NM 87545, U.S.A. \\ (e-mail: rskoug@lanl.gov)
}

(Received 10 January 2006; accepted 11 September 2006; Published online 28 November 2006)

\begin{abstract}
We present a comprehensive survey of 230 interplanetary CMEs (ICMEs) during 1995-2004 using Wind and ACE in situ observations near one AU, and examine the solar-cycle variation of the occurrence rate, shock association rate, scale size, velocity change, and other properties of ICMEs. The ICME occurrence rate increases (from 5 in 1996 to 40 in 2001) with solar activity; and $66 \%$ of all ICMEs occurred with shock(s). A compound parameter, the total pressure perpendicular to the magnetic field (Pt), i.e., the sum of magnetic and perpendicular plasma thermal pressures, assists us in effectively distinguishing ICMEs from other solar-wind structures such as stream interactions, and in quantifying the interaction strength. We interpret the characteristic signatures of the $P t$ temporal variation in terms of the inferred distance perpendicular to the flow to the center of the obstacle. Group 1 includes events that appear to be traversed near the ICME center, showing an apparent enhanced central $P t$; Group 3 represents ICMEs passed far away from the center, displaying a rapid rise and then gradual decay in $P t$; and Group 2 includes events with intermediate signatures. About $36 \%$ of 198 classifiable ICMEs are Group 1 events, consistent with the conventional wisdom that at one AU a magnetic cloud is found during crossings of only $~ 1 / 3$ of ICMEs. Our set of Group 1 ICMEs and the set of magnetic clouds from other researchers have significant overlap and a similar solar-cycle dependence. The rough decline of the Group 1 fraction as solar activity increases, is consistent with rough increases of scale size, shock percentage, and peak $P t$. These results call into question the need to have different mechanisms to create differently appearing ICMEs. Rather it is possible that all ICMEs have a central flux rope that is traversed about $33 \%$ of the time, but in the majority of cases is missed by the spacecraft.
\end{abstract}

Electronic Supplementary Material Supplementary material is available for this article at http://dx.doi.org/10.1007/s11207-006-0133-2 


\section{Introduction}

Coronal mass ejections (CMEs), seen in light scattered from enhanced electron densities in the solar corona (e.g. Gosling, Pizzo, and Bame, 1973; Gosling, 1990 and references therein), are transient events with large amounts of material ejected from the solar atmosphere (e.g., Hundhausen, 1988; Kahler, 1988). They play an important role in the long-term evolution of the corona (Hundhausen, 1999). Interplanetary CMEs (ICMEs), i.e., the interplanetary manifestations of CMEs, have been studied since the early decades of solar wind observations, as reviewed by Gosling (1990), Neugebauer and Goldstein (1997), and Rust (1999). Figure 1 is a simplified paradigm of an ICME, showing that the ICME frequently has a leading shock (or shock wave) and a well-formed, perhaps force-free flux rope at the center of the disturbance. In actuality by one AU, when the magnetic stresses are weaker relative to those in the plasma, the flux rope could deviate from the depicted dipolar shape and may not have such a circular cross section any more.

Since near the Earth, the density enhancement that defines a CME back at the Sun is generally not so evident, the identification of ICMEs is usually based on patterns of change in other properties of the magnetized plasma: a stronger than ambient magnetic field, rotating magnetic field (e.g., Hirshberg and Colburn, 1969; Burlaga et al., 1981; Klein and Burlaga, 1982), declining velocity (e.g., Klein and Burlaga, 1982; Russell and Shinde, 2003), low $\beta$ (ratio of the plasma thermal pressure to the magnetic pressure), abnormally low proton temperature (e.g., Gosling, Pizzo, and Bame, 1973; Richardson and Cane, 1995), low electron temperature (e.g., Montgomery et al., 1974), bidirectional solar wind electron strahls (BDEs) (e.g., Zwickl et al., 1983; Gosling et al., 1987), plasma-compositional anomalies (e.g., Hirshberg, Bame, and Robbins, 1972; Bame

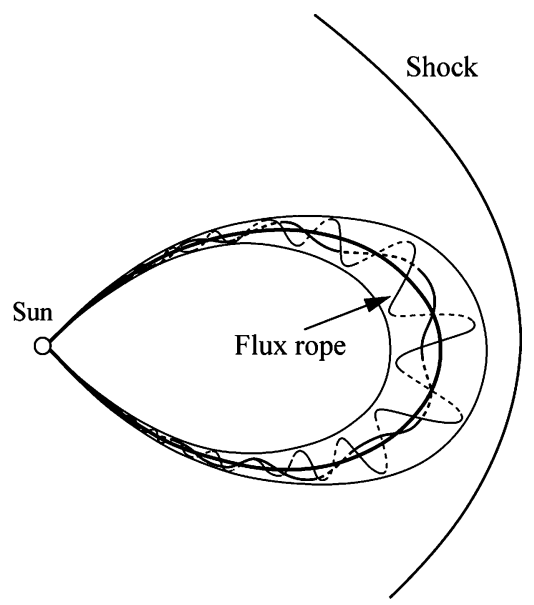

Figure 1. Simplified paradigm of Interplanetary Coronal Mass Ejection (ICME). 
et al., 1979; Ipavich et al., 1986; Goldstein, Neugebauer, and Clay, 1998), abnormal charge state of ions (e.g., Lepri et al., 2001), energetic particle signatures (e.g., Morrison, 1956; Palmer, Allum, and Singer, 1978; Marsden et al., 1987; Richardson, Cane, and von Rosenvinge, 1991) and others. None of these features appears to be unique to ICMEs or by itself a sufficient condition to identify an ICME (e.g., Gosling, 1997; Neugebauer and Goldstein, 1997). Furthermore, some of these parameters are not consistently available.

Because the above signatures of ICMEs arise from different physical conditions, such as plasma heating near the Sun during CME formation, expansion in the solar wind, and large-scale field structures, which may be rooted at the Sun, or reconnect with the interplanetary magnetic field (IMF), they may not occur exactly concurrently (e.g., Richardson and Cane, 2005). In fact, any of these characteristics could be missing and, if the others were present, some observers would argue for the presence of an ICME. For instance, some ICMEs or parts of ICMEs lack a BDE signature (e.g., Gosling, Birn, and Hesse, 1995; Shodhan et al., 2000); on the other hand, not only ICMEs, but shocks near the co-rotating interaction regions (CIRs) and magnetic connection of the IMF with planetary bow shocks, can also cause BDEs (e.g., Ogilvie, Scudder, and Sugiura, 1971; Feldman et al., 1973, 1982, 1983; Gosling et al., 1993; Steinberg et al., 2005). Also, some ICMEs lack a helium abundance enhancement (e.g., Zwickl et al., 1983; Phillips et al., 1995). So, based on varied criteria, several research groups have compiled lists of ICMEs, which are somewhat different from each other, e.g., Larson (http://sprg.ssl.berkeley.edu/ davin/clouds/cloud_list.html); Lepping (http://lepmfi.gsfc.nasa.gov/mfi/mag_cloud_publ.html); Cane and Richardson (2003); Liu, Richardson, and Belcher (2005); Russell and Shinde (2005).

The magnetic clouds (MCs) form a specific subset of ICMEs, and they are characterized by a low $\beta$ and by large coherent internal magnetic field rotations through a relatively large angle (Burlaga et al., 1981; Klein and Burlaga, 1982; Lepping, Jones, and Burlaga, 1990; Burlaga, 1991). Gosling (1990) concluded that about $30 \%$ of ICMEs at one AU exhibited magnetic flux ropes. However, it is not possible to show that $70 \%$ of ICMEs at one AU in fact lack a flux rope, because of the limitation of single-point observations. From the observations at one AU during solar cycles 20-21, 23 and also of Helios 1 and 2 at $0.3-1.0$ AU, Richardson and Cane (2004) reported a rough decrease in the MC fraction as the solar activity level increases. Different mechanisms have been proposed to form ICMEs, but because of the dominant role of the magnetic field in the eruption process through the conversion of magnetic energy into kinetic energy, modelers have not yet been able to devise a process that could produce a CME without an embedded flux rope (Riley et al., 2006).

So, it is possible that ICMEs all contain a well-defined flux rope close to the Sun (Marubashi, 1997), but that some flux rope signatures have weakened as the ICME evolves on its way to one AU (e.g., Osherovich and Burlaga, 1997). Alternatively, ICMEs may continue to contain identifiable flux ropes out to one $\mathrm{AU}$, but some 
of them are traversed far from the central flux rope where the flux rope can not be detected (e.g., Jian et al., 2005a; Riley et al., 2006). We address this proposition in detail below.

It is very appropriate to undertake this comprehensive study of a solar cycle of ICME activity at the present time. First, the Wind and Advanced Composition Explorer (ACE) spacecraft have just now obtained a sufficiently long data set to enable such a study to be undertaken. Second, we stand at the threshold of a new set of such data from the Solar-Terrestrial Relations Observatory (STEREO) mission, for which this study could aid in the interpretation. By determining the solar-cycle dependence of ICMEs we can place the early STEREO data at solar minimum into better context. Third, this study provides a baseline with which to compare data taken at distances closer to and further from the Sun, studies which we plan to undertake in the near future.

\section{Total Perpendicular Pressure}

Despite some well-developed physical models, some of which include launching CMEs and following them to Earth (e.g., Odstrcil and Pizzo, 1999a,b,c; Linker et al., 2003; Riley et al., 2003; Manchester et al., 2004), there is no thorough understanding of key aspects of CMEs, specifically, how they are initiated in the solar corona, and how they evolve to produce the signatures appearing in the in situ observations (Linker et al., 2003). It may be possible to improve our understanding with a new approach to choosing the plasma and field parameters to describe ICMEs, by moving away from specific measurements dictated by instrument outputs to a more physically-based parameter that controls the dynamics of the plasma.

Occasionally such a physically-based parameter, total pressure, has been used to characterize typical ICMEs (e.g., Gosling et al., 1987, 1994; Gosling, 1990), but it has not been utilized in a comprehensive study. Since the magnetic field does not exert a pressure force parallel to the field, the total perpendicular pressure (Pt), the sum of the magnetic pressure and plasma thermal pressure perpendicular to the magnetic field $\left[B^{2} /\left(2 \mu_{0}\right)+\sum_{j} n_{j} k T_{\text {perp }, j}\right.$, where $j$ represents proton, electron and $\alpha$ particle] is the key pressure component in determining the evolution of these magnetic structures (Russell, Shinde, and Jian, 2005).

If magnetic field lines are straight (no magnetic curvature force), $P t$ should tend to be slowly varying in the absence of the interaction with an obstacle, because unbalanced compressions lead to propagating waves that smooth the pressure profile. If there is a collision of the plasma with an obstacle, a force (gradient in the pressure) will occur that slows and deflects the plasma around the obstacle.

If field lines are not straight, the magnetic curvature force (twist in the rope) can contribute significantly to enhancing the magnetic field strength. It may even be self-balancing such as in a force-free flux rope. Hence, our simple pressure balance calculation (that implicitly assumes straight field lines) is insufficient to describe 
the force within a flux rope, and will overestimate the pressure. This should be noticeable in the tightly twisted field lines in the cores of ICMEs.

Our many case studies illustrate that much simpler signatures are found in $P t$ than in its constituent components for solar wind structures. The Pt is a good diagnostic of solar wind internal dynamics, be it an ICME or a stream interaction region (SIR), driven by fast wind overtaking slow wind (e.g., Gosling and Pizzo, 1999). In conjunction with the features of solar wind velocity, vector magnetic field and other parameters, $P t$ is an effective complementary parameter to distinguish ICMEs and SIRs. Moreover, it can quantify the interaction strength.

\section{Our Criteria, Data Set, and List of ICMEs}

In our study, the ICMEs are identified by eye from a combination of the $P t$ elevation, and the expected signatures of magnetic field and plasma addressed in Section 1, such as, the low proton temperature, a stronger than ambient magnetic field, a relatively quiet and smooth rotation in magnetic field, a helium abundance enhancement, BDE. However, none of these above characteristics, even the $P t$ enhancement, is a necessary condition when some other features of plasma and magnetic field are prominent. This explains why we have found some ICMEs with peak pressure $\left(P_{\max }\right)$ lower than $50 \mathrm{pPa}$. For some ambiguous events, we also check the SOHO Large Angle and Spectrometric Coronagraph (LASCO) (Brueckner et al., 1995) CME catalog (http://lascowww.nrl.navy.mil/daily_mpg/; http://cdaw.gsfc.nasa.gov/CME list/) to assure our identification.

Since it is hard to separate the magnetic obstacle from the magnetosheath region for some ICMEs, to be consistent and comparable, we set the boundary of all ICMEs associated with the outer distinct plasma and magnetic field discontinuities, often indicated by a rapid $P t$ jump. Therefore, our ICMEs include the shock (if it occurs), sheath pile-up region and the ejecta driver. For all the events with apparent magnetic obstacle structures, we give the start time of magnetic obstacle in the third column of the Appendix*, with its end time being the same as the whole event.

We use the Wind [SWE (Ogilvie et al., 1995) and MFI (Lepping et al., 1995), both in 93-second time-resolution] solar wind data set and the ACE [validated Level 2 of SWEPAM (McComas et al., 1998) and MAG (Smith et al., 1998), both in 64second time-resolution] solar wind data set. Because the measurement of electron temperature $\left(T_{e}\right)$, the ratio of number density of $\alpha$ to proton, $\alpha$ temperature, and the anisotropy of particle temperature, are not continuously available, we need to make some assumptions to calculate the $P t$.

*A tab-delimited text file of the Appendix is available as Electronic Supplementary Material at http://dx.doi.org/10.1007/s11207-006-0133-2 and is accessible for authorised users. It can be imported into MS Excel or other spreadsheet software. 
Electrons have high thermal conductivity, so their temperature generally varies in a small range and bears little correlation with other solar wind parameters (Newbury et al., 1998, and references therein). The average electron core temperature near one $\mathrm{AU}$ changes only from $123000 \mathrm{~K}$ around solar minimum to 144 $000 \mathrm{~K}$ near solar maximum (Issautier et al., 2005). Thus, we assume a constant solar wind electron perpendicular temperature of $130000 \mathrm{~K}$, which is also close to the median value of Newbury et al. (1998) from ISEE-3 measurements (August, 1978 - March, 1980). Because the $\alpha$ particles contribute a lesser amount to $P t$, we assume a constant $4 \%$ fraction of $\alpha$ particles by number with a temperature four times proton temperature. Without specific perpendicular temperatures for protons and $\alpha$ particles, we additionally assume that these temperatures are isotropic.

Wind and ACE both are close to the ecliptic plane at about one AU; ACE at the Lagrange $L_{1}$ point, while Wind was originally positioned in a sunward, multiple double-lunar swingby orbit with a maximum apogee of $250 R_{e}$ during its first two operation years, followed by a halo orbit at the Earth-Sun $L_{1}$ point, a distant prograde orbit with excursions of $300 R_{e}$ in the $Y_{\mathrm{GSE}}$ direction, and a trip to $L_{2}$, until now placed in a halo orbit about $L_{1}$. Hence, the use of the two spacecraft introduces a certain amount of variation in the timing of signatures of about one hour or so. However, ICMEs are large-scale spatial structures, with an average radial width of $\sim 0.25 \mathrm{AU}$ at the Earth's orbit (e.g., Klein and Burlaga, 1982), resulting in similar properties observed at the two spacecraft most of the time.

Because of its longer coverage, we derive our survey of ICMEs mostly from Wind. But in order to create as complete a list of ICMEs as possible, when Wind is too close to the Earth where the solar wind may be deflected by the Earth's magnetosphere, or it has data gaps or noisy data, we use ACE data, marked as "ACE" in the comments of the Appendix. From 1995 to 1997, Wind has data gaps or noise during only $3.8 \%, 7.6 \%$, and $3.7 \%$ of each year, respectively. We did not adjust our statistical results for these small outages as they are smaller than the expected statistical variability.

Using 1995-2004 Wind and 1998-2004 ACE solar wind data, we have identified 230 ICMEs to provide a comprehensive survey of ICMEs in the near-Earth solar wind (see Appendix), encompassing the end of solar activity cycle 22 and the rising, maximum and partially the declining phases of solar cycle 23 . The annual average ICME event number is 23 , but we do not rule out the possibility that we missed some events due to data gaps, noise and ambiguous ICME signatures. By examining the surrounding solar wind context, we also mark some hybrid events by a star in the Appendix.

The geomagnetic effects depend principally on the IMF (e.g., Russell and McPherron, 1973) and the solar wind velocity. So, we are most interested in these two quantities. Since the $P t$ in ambient solar wind is about $20-30 \mathrm{pPa}$, usually much less than the pressure of ICMEs, and in addition because we are concerned with the comparison between events, rather than an absolute value of pressure 
of each event, we just consider the magnitude of $P t$ rather than the difference between it and the background pressure. In the survey, we denote $\Delta P$ as the instantaneous change of the $P t$ across the discontinuity, $B_{\max }$ as the peak of $|\mathbf{B}|, R_{V}$ as the ratio of $V_{\max }$ to $V_{\min }, \Delta V$ as the change in the solar wind speed during each event. We emphasize that most ICMEs have a declining solar wind velocity (e.g., Klein and Burlaga, 1982; Russell and Shinde, 2003) with a negative value of $\Delta V$.

For a discontinuity simply indicated by $P t$, we examine the $V_{p}, N_{p}, T_{p}$, and $\mathbf{B}$ one by one, sometime also use the higher time-resolution Wind 3DP (Lin et al., 1995) and MFI, ACE SWEPAM and magnetometer data from CDAWeb, to verify if these parameters simultaneously vary and whether the discontinuity is a forward or reverse shock. In addition, we have compared our shock identification with the shock lists from Kasper (http://space.mit.edu/home/jck/shockdb/shockdb.html) and the ACE MAG and SWEPAM team (http://www-ssg.sr.unh.edu/mag/acel ACElists/obs_list.html) to confirm identifications.

\section{Three Groups of ICMEs}

It was evident early in the study that the temporal behavior of $P t$ in ICMEs displays characteristic patterns, that can be categorized into three groups (Jian et al., 2005a,b; Russell, Shinde, and Jian, 2005), illustrated by the three examples in Figures 2-4. These three figures have the same format, displaying several of the main parameters we use to characterize ICMEs. In the first three panels, we plot $B_{x} / B, B_{y} / B, B_{z} / B$, the direction cosines of IMF in the GSM coordinates. We do not use another common pair of angles, cone angle, arccos $\left(B_{x} / B\right)$ and clock angle, $\arctan \left(B_{y} / B_{z}\right)$ of IMF, because these two angles do not order the properties of ICMEs at one AU. Use of GSM coordinates will help in future studies of geoeffectiveness. The following panels are respectively $|\mathbf{B}|$ as the magnetic field magnitude, $V_{p}$ as the solar wind bulk velocity magnitude, $N_{p}$ as the proton number density, $T_{p}$ as the proton temperature, $\beta$ the ratio of plasma pressure to magnetic pressure, and total perpendicular pressure, $P t$, in the unit of pico-Pascal (pPa).

Figure 2 presents a typical Group 1 (G1) ICME, the shock and magnetosheath (the region between dashed lines $a$ and $b$ ) are followed by the magnetic obstacle (the region between dashed lines $b$ and $c$ ), $P t$ increases rapidly at the sheath and piles up to a central maximum in the later magnetic obstacle. We can observe such well-defined MCs as shown in Figure 2, in most G1 events, but not all G1 events have obvious shocks. Group 2 (G2) ICMEs have a rapid rise in $P t$, again, not necessarily a shock (a shock is not a required criterion for ICME identification), with a pressure plateau and a much later return to earlier lower pressure (Figure 3). Among G2 events, we can see some signatures of MCs, but they are less obvious than G1. In Group 3 (G3) ICMEs, the pressure profile usually rises rapidly and then 


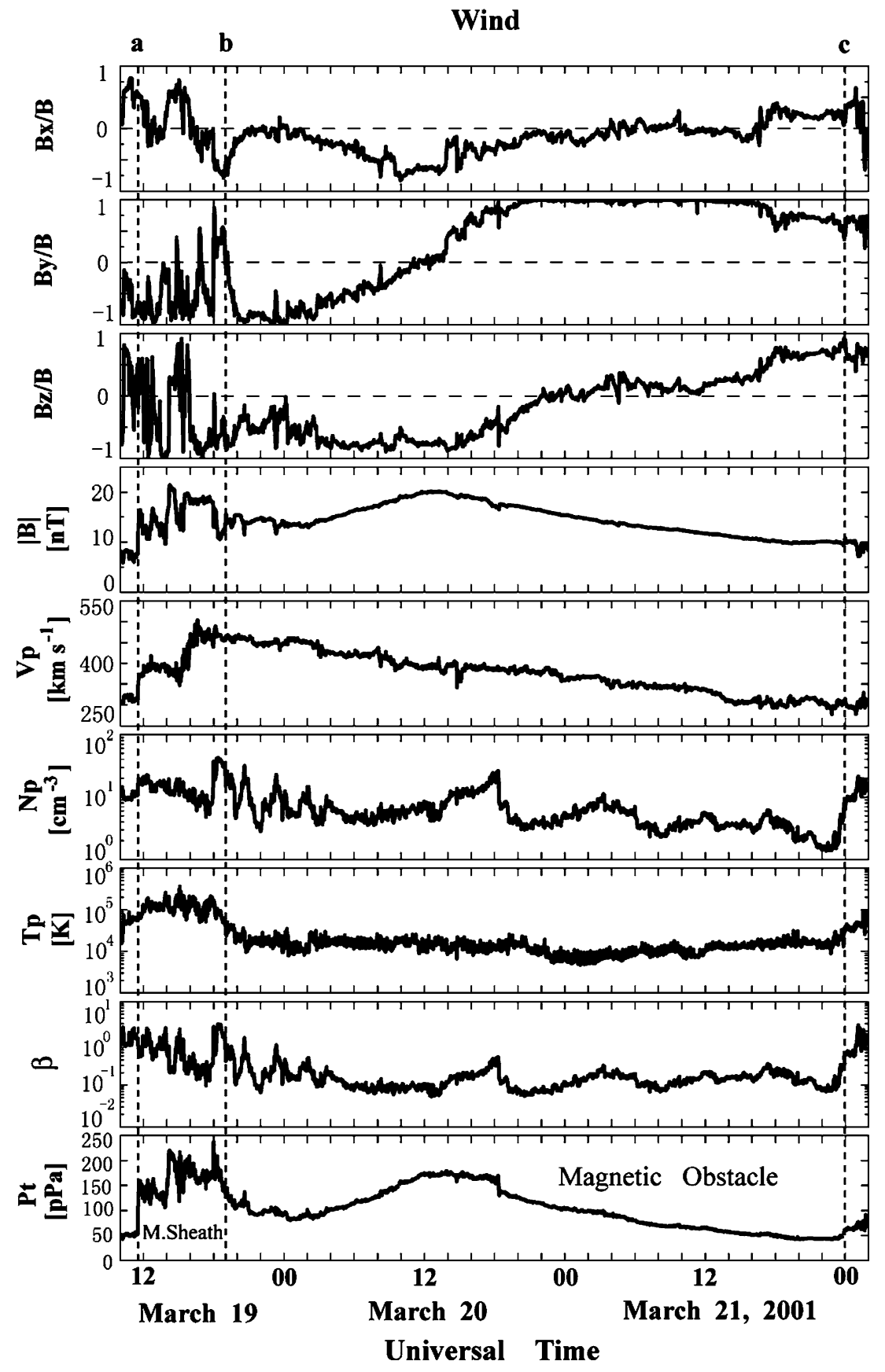

Figure 2. Group 1 ICME from Wind observation. From top to bottom: direction cosines of IMF in GSM coordinates, magnetic-field strength, solar-wind speed, proton density, proton temperature, $\beta$, and total perpendicular pressure. M. Sheath: magnetosheath, the interval between the dashed lines $a$ and $b$; magnetic obstacle, the region between dashed lines $b$ and $c$. 


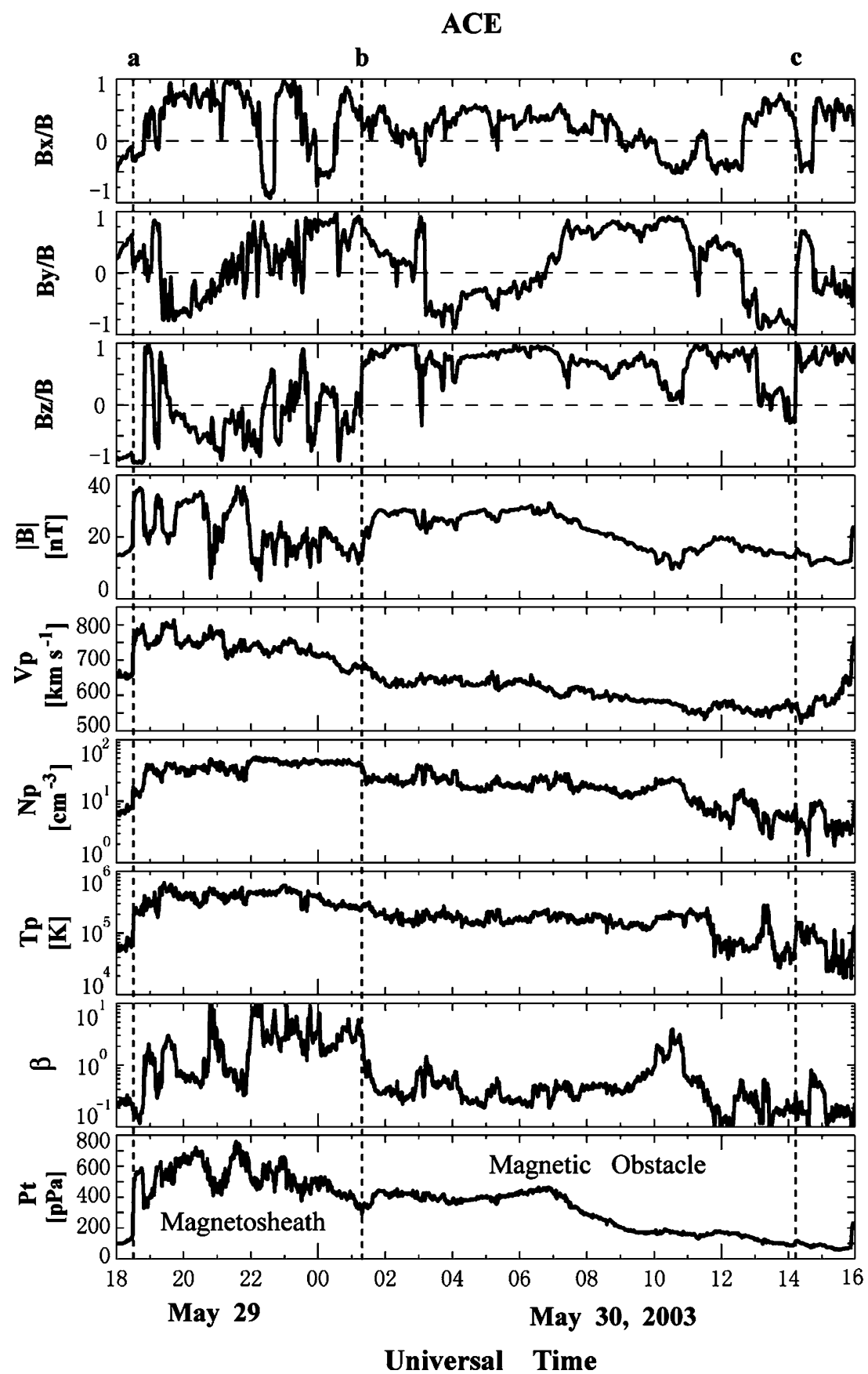

Figure 3. Group 2 ICME from ACE observation. Comments in the caption of Figure 2 apply. 


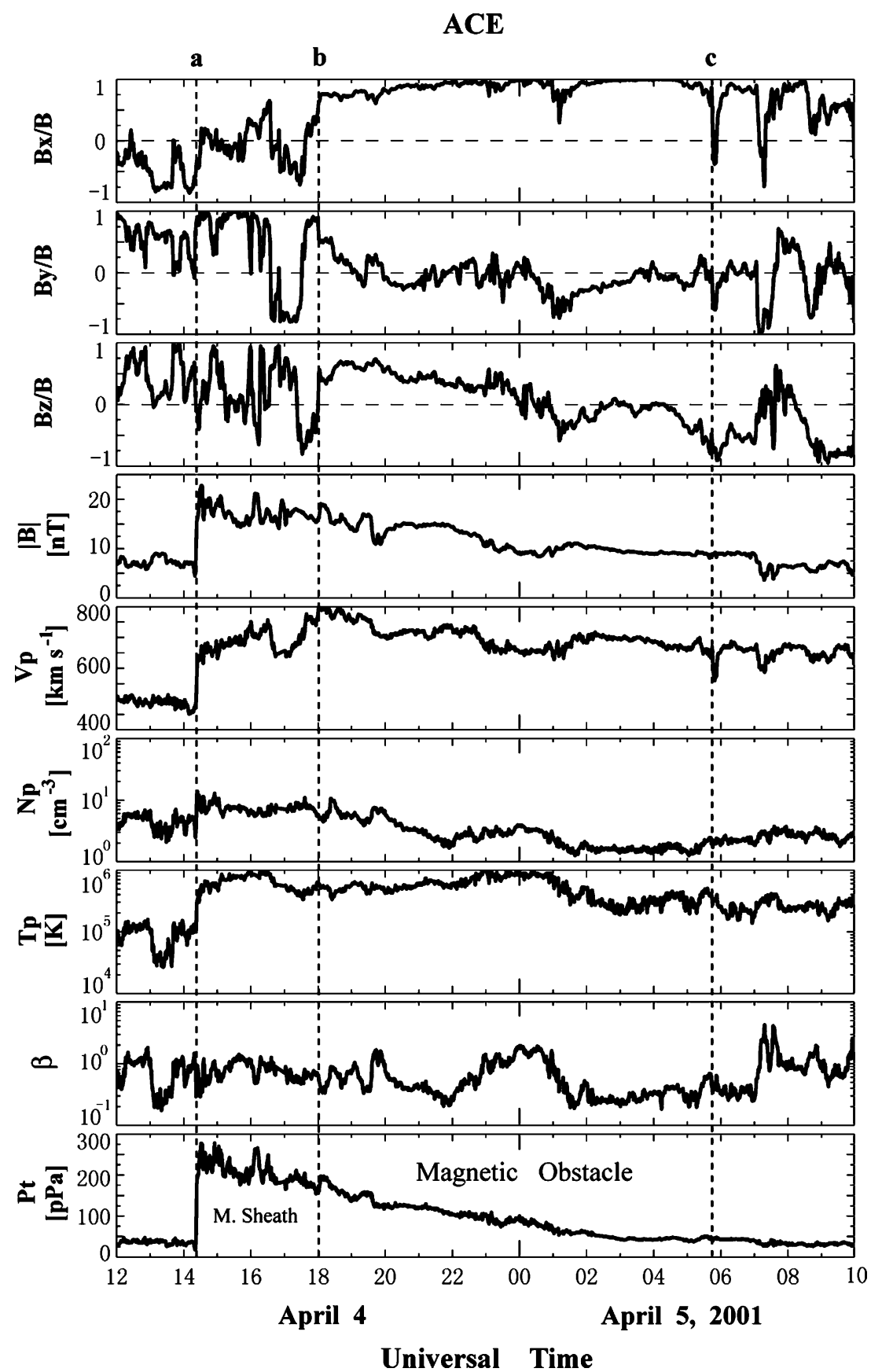

Figure 4. Group 3 ICME from ACE observation. Comments in the caption of Figure 2 apply. As a clear example of Group 3 ICMEs, we can still see the magnetic obstacle from the magnetic field measurements between dashed lines $b$ and $c$. But for $92 \%$ of the Group 3 events, magnetic obstacles can not be well discerned. 
gradually decays over hours or days. During such cases, the individual features of the MC, such as the stronger than ambient and rotating magnetic field, are usually not recognizable. Figure 4 shows a relatively clear G3 example, where we can still see traces of the magnetic obstacle from the magnetic field measurements. However, it is hard to discern the obstacle solely from the plasma observation and compound parameters $\beta$ and $P t$.

Hence, the presence of MC signatures is correlated with the Pt profiles. Considering the center of magnetic obstacle usually has larger pressure than in other regions, the G1 events appear to mark the passage of the ICME through or near the center of ICMEs, where we see the MC signatures. The G3 event with few MC signatures, appear to be just glancing encounters. Thus, the different patterns of the $P t$ profile may indicate different impact parameters of spacecraft relative to the center of ICMEs. If there is a flux rope in the center of ICME, the dynamic (expanding or just moving faster than the solar wind) ICMEs should create a disturbance in the ambient solar wind greater than the size of the embedded flux rope. Spacecraft that do not penetrate the central region of an ICME will not always see the simple MC structure drawn in Figure 1.

In Figure 5, we have drawn a flux rope in the place of the magnetosphere used as the obstacle to the flow in the Spreiter, Summers, and Alksne (1966) gasdynamic simulation of flow passing a blunt object. The contours show the density which we take as a rough proxy for the pressure. We are aware that the CME will undergo considerable distortion as it moves away from the Sun and may have complicated geometry by the time it reaches one AU (e.g., Odstrcil and Pizzo, 1999c; Riley et al., 2003), in contrast to the circularly symmetric flux rope as shown in Figure 5. We interpret our three groups of ICMEs as corresponding to different distances of the spacecraft passes through the ICME relative to the central flux rope (origin in Figure 5). These trajectories are marked in Figure 5 by arrows.

Nevertheless, the above sorting is idealized and the variation from Group 1 to 3 is a continuum with somewhat arbitrary definitions. For example, over the ten years of our study, $8 \%$ of the G3 ICMEs appear to traverse a part of a magnetic obstacle. The spacecraft may only pass a part of cloud, without seeing a complete cloud structure. We emphasize that the patterns form a continuum without sharp boundaries between the categories.

In contrast to the ICME $P t$ profiles, the SIRs usually have a peak with a slow increase and decrease of pressure on its two sides (e.g., Gosling and Pizzo, 1999; Jian et al., 2006), indicating the forces pushing outward to the two sides of the stream interface. However, many CMEs observed at the Sun are slow (e.g., Gosling et al., 1976), and they may be difficult to distinguish from SIRs just by their pressure profile, unless they have sufficient internal pressure and expansion to produce a shock. But from the plasma and magnetic field features, composition signatures (e.g., $\alpha$ particles abundance) and electron topology (BDEs), it is also possible to distinguish them. 


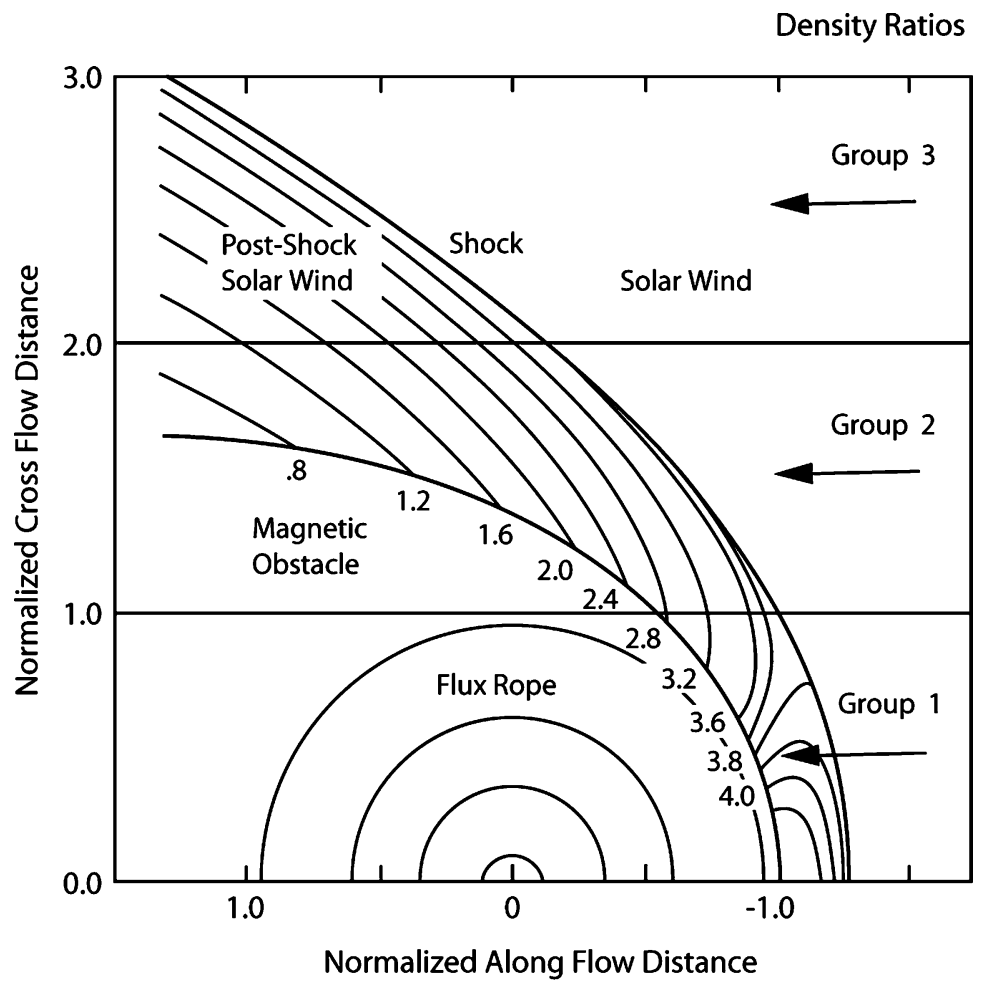

Figure 5. Interpretive sketch of ICME encounters using Spreiter, Summers, and Alksne (1966) gasdynamic simulation results. Group 1 events encounter the magnetic rope. Group 2 events encounter the ICME near the obstacle. Group 3 events catch the shock away from the obstacle. In reality, the magnetic rope is not circularly symmetric but distorted.

\section{Occurrence Rate of ICMEs}

The annual number of ICMEs from 1995 to 2004 is given in Table I, and also shown in panel (a) of Figure 6. The ICME occurrence rate varies greatly, from 5 in 1996, around solar minimum, up to 40 in 2001, near solar maximum (consistent with the largest halo CME number in 2001, halo CME number from SOHO LASCO CME catalog), increasing monotonically except for 1999 as the solar activity level increases. The unusually low occurrence rate in 1999 is also noted in Cane, Richardson, and St. Cyr (2000) and Cane and Richardson (2003), probably associated with an increase of co-rotating highspeed streams from low-latitude coronal holes and the restructuring of the nearecliptic solar wind in 1999 (e.g., Luhmann et al., 2002; Cane and Richardson, 2003).

We classify 198 ICMEs with clear $P t$ characteristics into three groups. Table I shows the number in each group of ICMEs as well as their relative occurrence rate. 


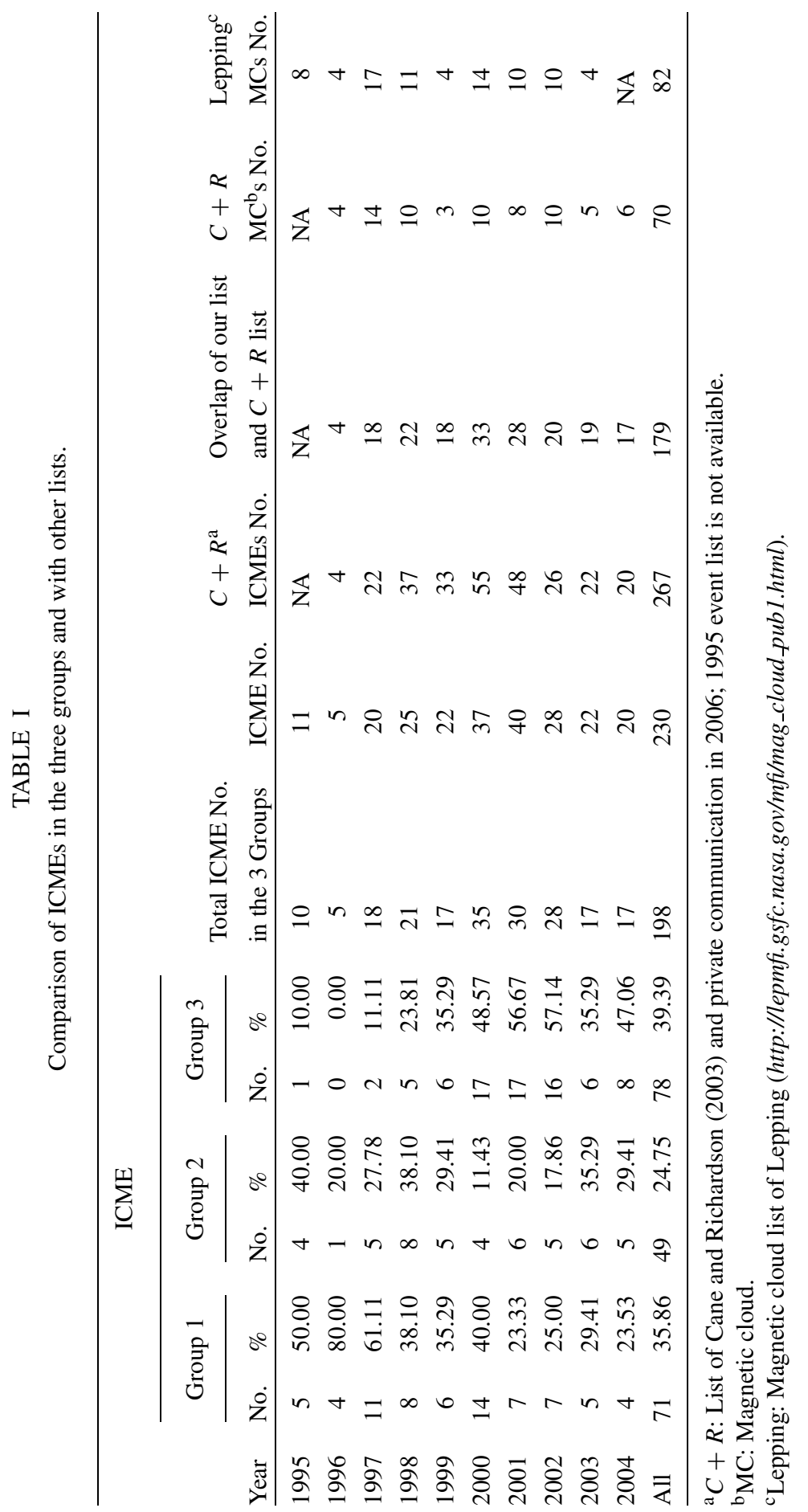



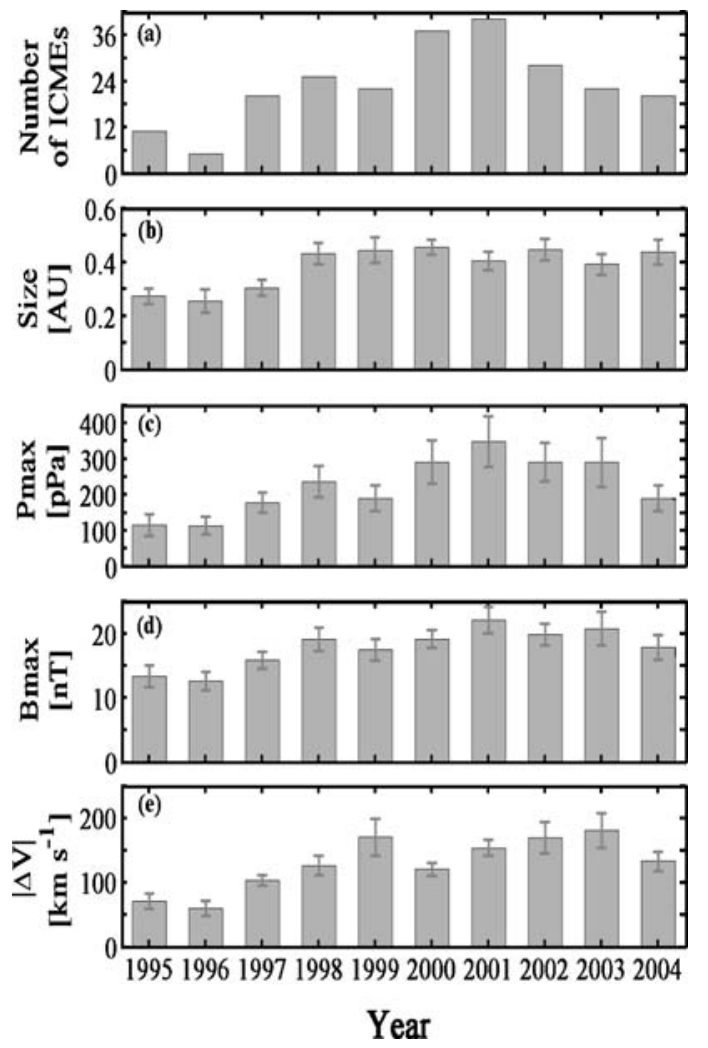

Figure 6. Annual statistics of some properties of ICMEs during the period 1995-2004. (a) Occurrence rates of ICMEs. (b) Scale size for each ICME. (c) Peak total perpendicular pressure. (d) Maximum magnetic field. (e) Absolute change in solar wind velocity during one event. The probable error of the mean is indicated.

Among them, there are $71 \mathrm{G} 1$ events, suggesting that on average $~ 36 \%$ of ICMEs are penerated by Wind or ACE near the center of the flux rope. This is consistent with the conventional wisdom that about 1/3 of ICME observations are encounters with MCs at one AU.

Figure 7 illustrates the trend of the percentages of the three groups ICMEs of all the classifiable ICMEs over 1995-2004. The extent of the vertical axis is the sum of the three fractions, which is $100 \%$ for each year. The occurrence rate of G1 ICMEs (dark gray) [see the left-hand scale], roughly decreases as solar activity strengthens, with a peak at solar minimum; while the occurrence rate of G3 ICMEs (light gray), [see the right-hand scale], has the opposite trend, overtaking the G1 occurrence rate in 2000-2002. This anti-correlation of G1 and G3 during the solar cycle is consistent with a stronger dynamic interaction of the ICME with the ambient solar wind around solar maximum (e.g., Riley et al., 2006) causing a larger region of disturbance around the central flux rope. This 


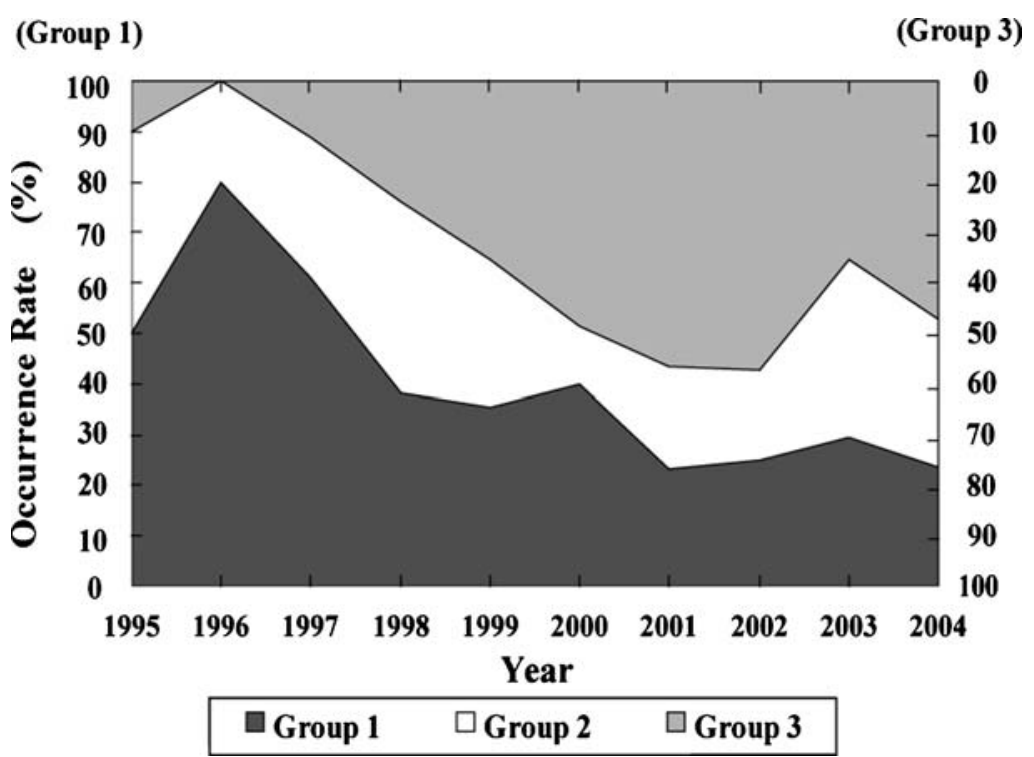

Figure 7. Occurrence rates of ICMEs in three groups during 1995-2004.

hypothesis is consistent with the rough increases of $P_{\max }$, scale size, and shock associations. The white region between the two gray areas represents the percentage of G2 ICMEs, without much variation over the 10 years, being smallest in 2000.

In addition, there are 32 events too complicated to be sorted into any of the three groups. Some of them are hybrid events of ICME and SIR or of more than one ICME, and others have some irregular pressure profiles, often with extremely low $P t$. We note that if they could be classified as members of one of the three groups, the statistics would change but slightly.

In Table I, we also list the number of our identified ICMEs and compare them with ICMEs identified by Cane and Richardson (2003, and private communication in 2006) (hereinafter referred to as CR). They did not classify ICMEs in the available 1995 data. The CR study used quite a few characteristics such as low proton temperature, reduced magnetic field fluctuations, and other criteria. We also list MCs identified by Lepping for each year from 1995 to 2003. The ICME rates given from the three research groups have similar solar-cycle dependence. But for the period $1998-$ 2000, CR find over ten events per year more than we do, possibly because they have also used Interplanetary Monitoring Platform (IMP 8) data and additional criteria. As they intended, this study should have produced the most liberal or inclusive list. We note that taking many signatures (e.g., solar energetic particles, cosmic ray, etc.) into account, will reduce the influence of the plasma and field parameters on the identifications. 


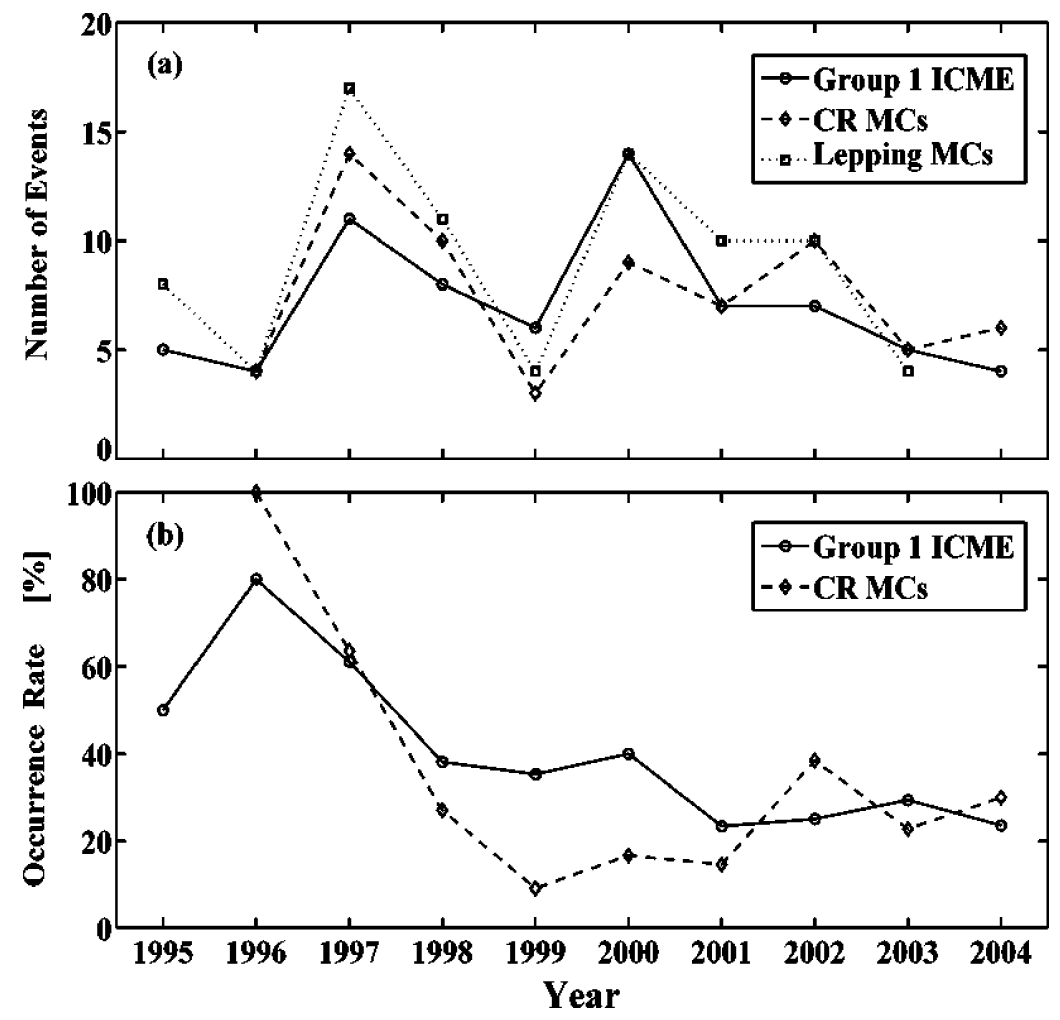

Figure 8. (a) Comparison of the number of events of our identified Group 1 ICME and magnetic clouds (MCs) identified by Cane and Richardson and by Lepping. (b) Occurrence rates of Group 1 ICMEs and MCs identified by Cane and Richardson.

We also give the yearly number of overlap events of our study and the CR list in the third-to-last column of Table I. In all from 1996-2004, 179 events, accounting for about $82 \%$ of our ICMEs are in the CR list, while approximately $67 \%$ of their events are in our list. These two statistics indicate that the techniques are different, rather than simply that one is more liberal than the other.

There is an MC quality index in the CR list $(0,1$, and 2 indicating increasing quality of the MC). Approximately, $63 \%$ of our G1 ICMEs are also in their MC $=2$ list, and $26 \%$ of our G1 events are in $\mathrm{MC}=1$ list. Conversely, 18 (accounting for $28 \%$ ) of their $\mathrm{MC}=2$ events end up in our G2, and $7 \mathrm{MCs}(11 \%)$ are in G3. In addition, about 41 events (62\%) of our G1 ICMEs were in Lepping's MC table, and on the other hand, 17 cases (39\%) of Lepping's MC list end up in our G2, and $8 \mathrm{MCs}(11 \%)$ are in G3. Considering that our grouping and CR's MC index are both continua with somewhat subjective definitions, the overlap of our G1 ICMEs and other groups' MCs is significant, and the low number of MC signatures in our G2 and G3 lists indicates that these independent approaches are finding consistent results. 
Figure 8(a) compares the solar-cycle variations of the number of G1 ICMEs (solid line marked by circles), MCs identified by CR (dashed line marked by diamonds), MCs identified by Lepping (dotted line marked by squares). We can see they have similar solar-cycle dependence. Figure 8(b) shows the percentages of G1 ICMEs relative to all the ICMEs in our study (solid line marked by circles) and MCs relative to all the ICMEs identified by CR (dashed line marked by diamonds). The former is small in 2001-2004, while the latter reaches minimum around solar maximum (Richardson and Cane, 2004). The two trends are similar, except for two places on the curve. There is no CR value for our first year 1995. If we use their identified number of ICMEs and MCs for 2004 as indicative of what they would have found in 1995, the curves would be very similar through solar minimum. But we are aware that there is a major difference between 1999 and 2000, where CR identified 30\% more ICMEs than we did, but fewer MCs than we did. This again suggests a difference in our criteria for ICME identifications or G1-MC association may not be completely accurate. Nevertheless, their overall result that the portion of magnetic clouds drops at solar maximum, is confirmed by our analysis.

\section{Solar-Cycle Variation of Properties of ICMEs}

Table II lists the average characteristics of ICMEs observed from 1995-2004. It gives the annual number of ICME events, the number and percentage of events

TABLE II

ICME statistics.

\begin{tabular}{|c|c|c|c|c|c|c|c|}
\hline Year & $\begin{array}{l}\text { ICME } \\
\text { No. }\end{array}$ & $\begin{array}{l}\% \text { with } \\
\text { shock }\end{array}$ & $\begin{array}{l}\% \text { with } \\
\text { shock }\end{array}$ & $\begin{array}{l}\left\langle P_{\max }\right\rangle \\
\left(\delta P_{\max }\right)^{\mathrm{a}}\end{array}$ & $\begin{array}{l}\left\langle B_{\max }\right\rangle \\
\left(\delta B_{\max }\right)\end{array}$ & $\begin{array}{l}\left\langle R_{V}=V_{\max } /\right. \\
\left.V_{\min }\right\rangle\left(\delta R_{V}\right)\end{array}$ & $\begin{array}{l}\langle|\Delta V|\rangle \\
(\delta|\Delta V|)\end{array}$ \\
\hline 1995 & 11 & 5 & 45.5 & $114.09(30.40)$ & $13.34(1.65)$ & $1.22(0.04)$ & $71.36(11.37)$ \\
\hline 1996 & 5 & 0 & 0.0 & $113.00(24.27)$ & $12.58(1.42)$ & $1.18(0.04)$ & $60.20(12.16)$ \\
\hline 1997 & 20 & 8 & 40.0 & $176.90(27.67)$ & $15.79(1.35)$ & $1.31(0.03)$ & $102.90(7.90)$ \\
\hline 1998 & 25 & 17 & 68.0 & $235.00(42.51)$ & $18.98(1.81)$ & $1.34(0.03)$ & $126.12(14.50)$ \\
\hline 1999 & 22 & 15 & 68.2 & $189.18(34.89)$ & $17.38(1.70)$ & $1.44(0.08)$ & $169.64(28.73)$ \\
\hline 2000 & 37 & 28 & 75.7 & $289.43(59.69)$ & $19.06(1.41)$ & $1.29(0.02)$ & $120.57(9.81)$ \\
\hline 2001 & 40 & 31 & 77.5 & $346.66(70.75)$ & $21.94(2.07)$ & $1.36(0.03)$ & $153.37(12.87)$ \\
\hline 2002 & 28 & 23 & 82.1 & $288.68(53.54)$ & $19.71(1.65)$ & $1.44(0.06)$ & $169.50(24.13)$ \\
\hline 2003 & 22 & 13 & 59.1 & $288.05(67.52)$ & $20.64(2.57)$ & $1.37(0.05)$ & $179.76(26.33)$ \\
\hline 2004 & 20 & 11 & 55.0 & $188.90(35.96)$ & $17.75(1.90)$ & $1.33(0.03)$ & $132.65(14.93)$ \\
\hline All & 230 & 151 & 65.7 & 251.59 (19.39) & $19.27(0.71)$ & $1.35(0.01)$ & $138.89(6.18)$ \\
\hline Max & 40 & 31 & 82.1 & 2100 & 80 & 2.96 & 615 \\
\hline Min & 5 & 0 & 0.0 & 24 & 3.5 & 1.07 & 30 \\
\hline
\end{tabular}

a $\delta$ Presents the probable error of the mean for the corresponding parameter. 
with shocks, the average $P_{\max }, B_{\max }, R_{V}$, absolute difference of $V_{p}$ magnitude $(|\Delta V|)$ as well as their probable errors of the mean during 1995-2004. The bottom three rows list the average, maximum and minimum of these properties among all events. Averaged over the ten years, $P_{\max }$ is $252 \pm 19 \mathrm{pPa} ; B_{\max }$ is $19.3 \pm 0.7 \mathrm{nT} ; R_{V}$ is $1.35 \pm 0.01$; and $|\Delta V|$ is $139 \pm 6 \mathrm{~km} \mathrm{~s}^{-1}$, where the uncertainty is the probable error of the mean. The $R_{V}$ and $|\Delta V|$ are smaller than the corresponding values of SIRs, $1.66 \pm 0.02,230 \pm 5 \mathrm{~km} \mathrm{~s}^{-1}$ (Jian et al., 2006).

In all, 65.7\% of ICMEs drive shocks at one AU. Ten of the ICMEs at one AU began with two or more forward shocks, of which we count as only one forward shock herein. Besides some hybrid events, the other ICMEs are isolated events, but they indeed occur with more than one shock, and these shocks are usually nearly in contact. The fraction of shocks varies roughly in phase with solar activity at one AU, peaking at $82 \%$ in 2002. The shock association rate of ICMEs at one AU is much higher than the rate from Pioneer Venus Orbiter (PVO) observations (1979-1988) at $0.72 \mathrm{AU}$, where even the highest annual rates are still less than 30\% and these occur in the declining phase (Lindsay et al., 1994). While these measurements were made in different solar cycles, they suggest that most ICMEs shocks arise from 0.72 to $1.0 \mathrm{AU}$. We will address this issue in a future study.

We have found only one single reverse shock, and it is associated with a hybrid event. In all, only three events occurred with forward-reverse shock pairs, and they happened in 2000-2001, around solar maximum, with the forward shocks much stronger than the corresponding reverse ones. None of them is associated with overexpansion, verifying that shock pairs associated with overexpansion (Gosling et al., 1994) have never been observed at low heliographic latitudes at any heliocentric distance (Gosling et al., 1995).

The five panels in Figure 6 respectively display the solar-cycle variations of the occurrence rate, scale size, $P_{\max }, B_{\max }$ and $|\Delta V|$ of ICMEs from 1995 to 2004, where the error bar is the corresponding probable error of the mean. The ICME annual average duration has no clear solar-cycle dependence, varying from 5.5 to 94 hours, with an average of $35 \pm 1$ hours.

We use the mean of $V_{\max }$ and $V_{\min }$ as the average velocity, and estimate the scale size of each ICME by the product of average velocity and duration. The size varies from 0.08 to $1.08 \mathrm{AU}$, and has an average of $0.41 \pm 0.01 \mathrm{AU}$. The annual average size is larger around the solar maximum, except for 2001, again suggesting the ICMEs may affect a larger region during high solar activity. The shock-to-magnetic obstacle sheath region was found on average to be $0.16 \mathrm{AU}$, with a most probable thickness of $0.13 \mathrm{AU}$, in an earlier study by Gosling et al. (1987). Our result is larger than the size (0.25 AU) found by Klein and Burlaga (1982) two decades ago. This difference is probably due to our inclusion of the pile-up/magnetosheath region in our size estimate.

The value of $P_{\max }$, i.e., the interaction of ICMEs with ambient solar wind or within the ICMEs, increases roughly with the solar activity level, except for 1999. 

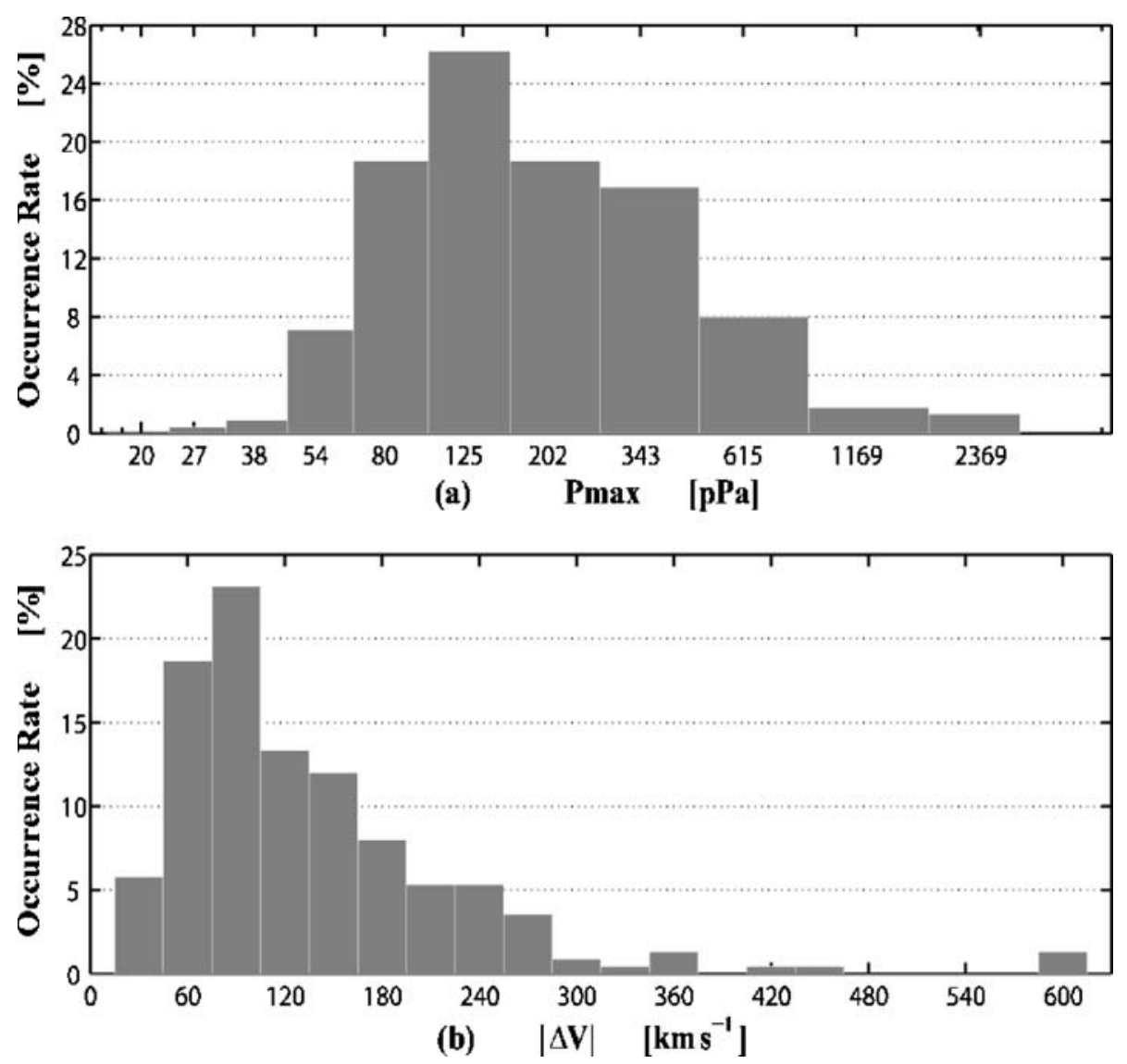

Figure 9. Probability distribution: $P_{\max }$ and $|\Delta V|$ of ICMEs (1995-2004).

It varies greatly over the ten years, from the minimum $(113 \pm 24 \mathrm{pPa})$ in 1996 to the maximum $(347 \pm 71 \mathrm{pPa})$ in 2001 . The maximum value is up to three times larger than the minimum. During $2000-2003$, the variability is over $50 \mathrm{pPa}$, associated with the large variability of $P_{\max }$ near solar maximum. The value of $B_{\max }$ has a similar solar-cycle variation, with the maximum about twice as large of the minimum. The similarity is expected, because ICMEs are low- $\beta$ structures and the magnetic pressure makes the largest contribution to $P t$.

The annual averages of $R_{V}$ and $|\Delta V|$ also change much through the ten years. They both reach a minimum around solar minimum and have some large values around solar maximum. Unexpectedly, the two largest $R_{V}$ values occur in 1999 and 2002, and so does $|\Delta V|$.

The two panels $(a$ and $b$ ) in Figure 9 individually show the probability distributions of $P_{\max }$ and $|\Delta V|$ of these relatively dynamically active ICMEs, one on a quasi-logarithmic scale (to distribute the data well across the bins, we use bin values successively raised by the power 1.1), and the other distribution on a 
linear scale. They are both almost centrally distributed. The value of $P_{\max }$ varies extensively from 24 to $2100 \mathrm{pPa}$, and is distributed mostly around $125 \mathrm{pPa}$, where about $26 \%$ of ICMEs fall. The $|\Delta V|$ varies from 30 to $615 \mathrm{~km} \mathrm{~s}^{-1}$, and $\sim 41 \%$ of the 230 ICMEs have the $|\Delta V|$ falling between 45 and $105 \mathrm{~km} \mathrm{~s}^{-1}$. Moreover, the probability distribution of $|\Delta V|$ has a long tail, caused by some ICMEs with quite large velocity variation.

\section{Conclusions}

Total perpendicular pressure $(P t)$ in the interplanetary medium near one AU has a simple temporal variation, smooth except for shocks, quiet in contrast to the variations of its individual components. This feature has assisted us greatly in identifying ICMEs.

From 1995-2004 Wind and ACE solar wind data, we identify 230 ICMEs, and $66 \%$ of them occur with shocks, three associated with forward-reverse shocks pairs. The occurrence rates, scale size, $P_{\max }, B_{\max }$, and $|\Delta V|$ of ICMEs follow the solar-activity variation, while the duration has no clear solar-cycle dependence. The $P_{\max }$ and $|\Delta V|$ both have a broad probability distribution, with the mean values of $252 \pm 19 \mathrm{pPa}$ and of $139 \pm 6 \mathrm{~km} \mathrm{~s}^{-1}$ respectively. The average size of our ICMEs is $0.41 \pm 0.01 \mathrm{AU}$, and the maximum annual average is about twice the minimum.

Our comprehensive survey of one AU observation of ICMEs can provide a baseline to compare data taken at other heliocentric distances, to further understand the ICME evolution in the heliosphere. It will also help interpret the coming STEREO data, especially in putting STEREO's solar minimum observations in the context of the overall expected solar-cycle variation.

The ICME $P t$ temporal profiles have three characteristic patterns, which are correlated with the observed MC signatures. Corresponding to Group 1, 2, and 3 ICMEs, the $P t$ profile following the shock and/or sheath increases has a central pressure maximum, or a steady plateau, or a gradual decay, respectively. We interpret the three groups of $P t$ profiles as being associated with different distances of approach to the causative central flux rope in each ICME. The absence of an observed flux rope or cloud in the majority of ICMEs does not imply the absence of such a flux rope in the center of the structure.

This interpretation is supported from three aspects in this study. (1) Averaged over ten years, $\sim 36 \%$ of 198 classifiable ICMEs are G1 events, consistent with the general wisdom that about 1/3 of ICME observations at one AU are encounters with magnetic clouds. (2) Our G1 ICMEs and MCs identified by other investigators, mostly overlap and have similar solar-cycle dependence, suggesting that MCs are mainly the G1 ICMEs, which spacecraft pass through the center and observe the expected MC signatures. (3) The fractions of G1 and G3 ICMEs are anti-correlated, and the percentage of G1 (flux rope encountered) roughly decreases as the solar activity enhances, comfirmed by earlier work of Richardson and Cane (2004). 
The last variation can have contributions from several factors. The interaction of the flux rope with the ambient solar wind or other ICMEs clearly gets stronger as solar activity strengthens, consistent with rough solar-cycle trends of shock association rate, scale size and $P_{\max }$ we have found. Thus, the size of the disturbed region relative to the size of the disturbing central obstacle will change with the solar cycle. Around solar maximum, the latitudinal spread of CME central axes is bigger (Hundhausen, 1993), and a larger fraction of CMEs originate from the mid- or high-heliolatitude of the Sun (e.g., Riley et al., 2006), resulting in a smaller probability of spacecraft in the ecliptic plane passing through the center of ICMEs.

The launch of STEREO will enable multi-spacecraft observations that will allow us to make two or more (using ACE or Wind) cuts through ICMEs at varying distances from the center, enabling us to establish the ecliptic longitude variation of these structures and to test our hypothesis that all ICMEs may have central flux ropes, and that the ICME Pt signature depends on the impact parameter.

\section{Acknowledgements}

This work is supported by the IGPP branch at Los Alamos National Lab (LANL). We have used the Wind plasma and magnetic field data throughout. We thank the MIT and Goddard plasma team (A.J. Lazarus and K.W. Ogilvie), 3DP plasma team (R.P. Lin) and the magnetometer team (R.P. Lepping) for making these data available. We have incorporated ACE data in this study with. So we are grateful to the PIs of the plasma analyzer (D.J. McComas) and of the magnetometer (C.W. Smith) for making these data publicly available, and also thank J. Steinberg for collaboration in LANL. Work at Los Alamos was performed under the auspices of the U.S. Department of Energy, with financial support from the NASA ACE program. Moreover, to identify the shocks, we used the higher-time-resolution data in CDAWeb; we thank CDAWeb for making the data available. 


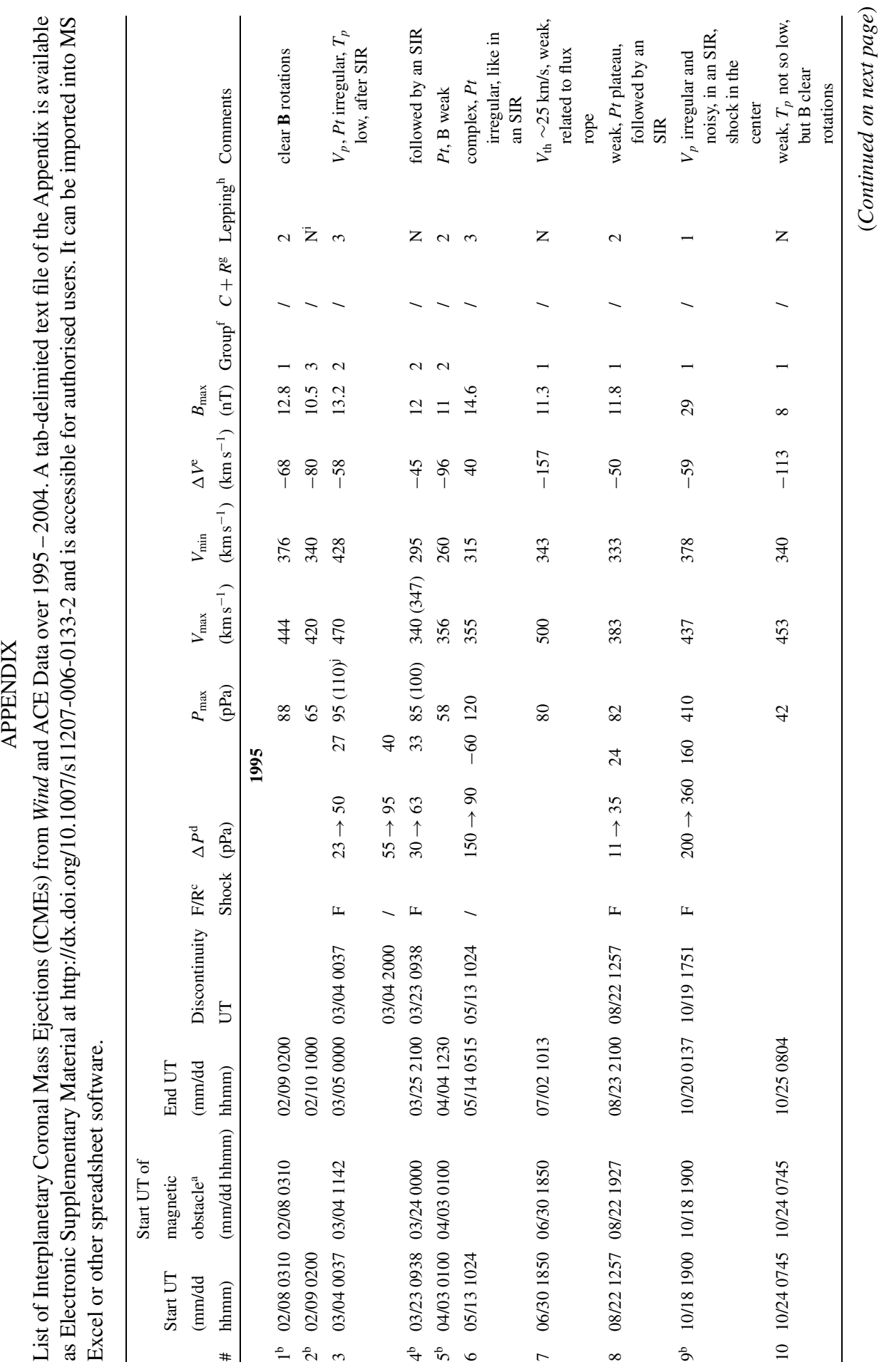


PROPERTIES OF INTERPLANETARY CORONAL MASS EJECTIONS

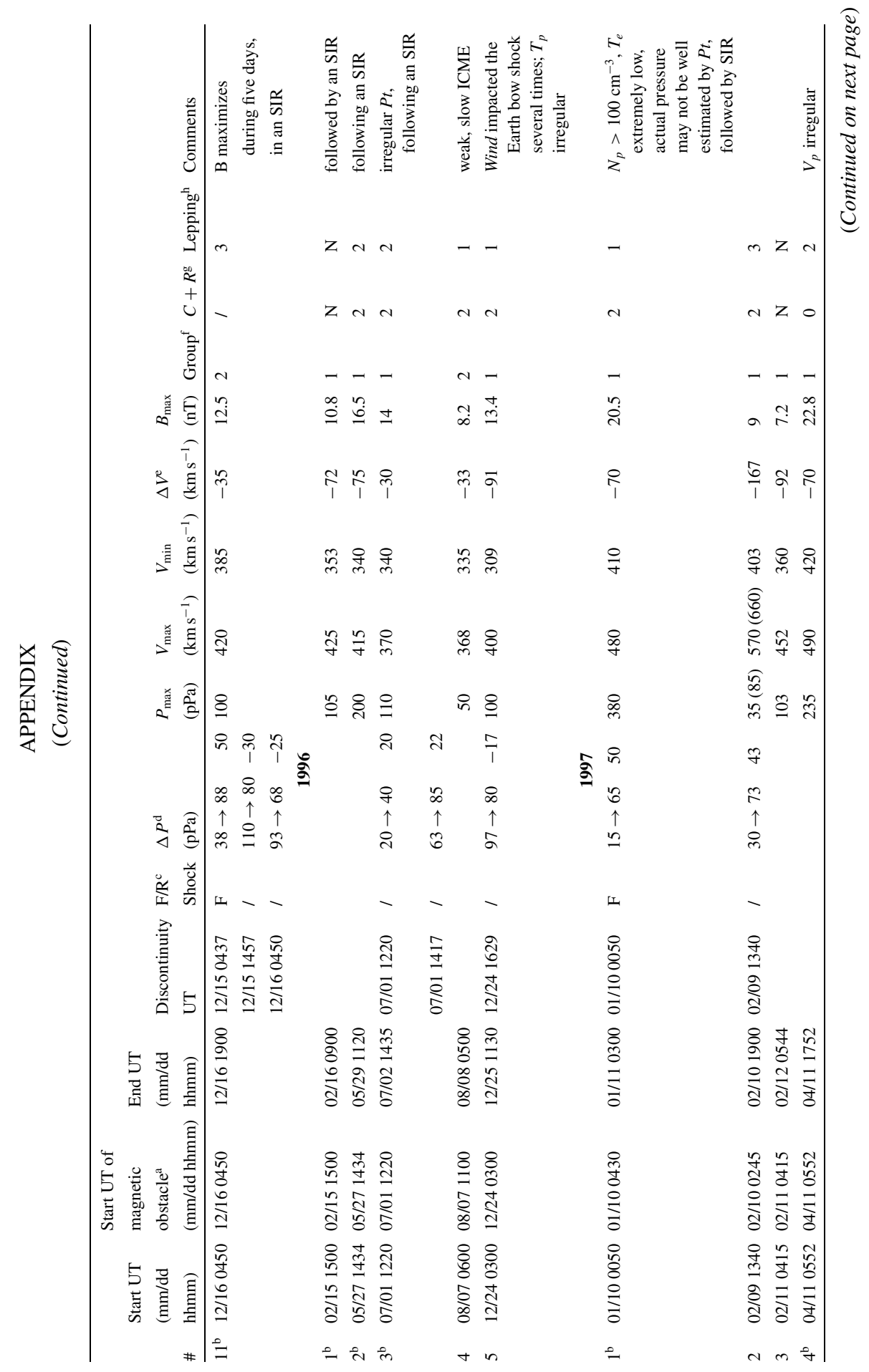




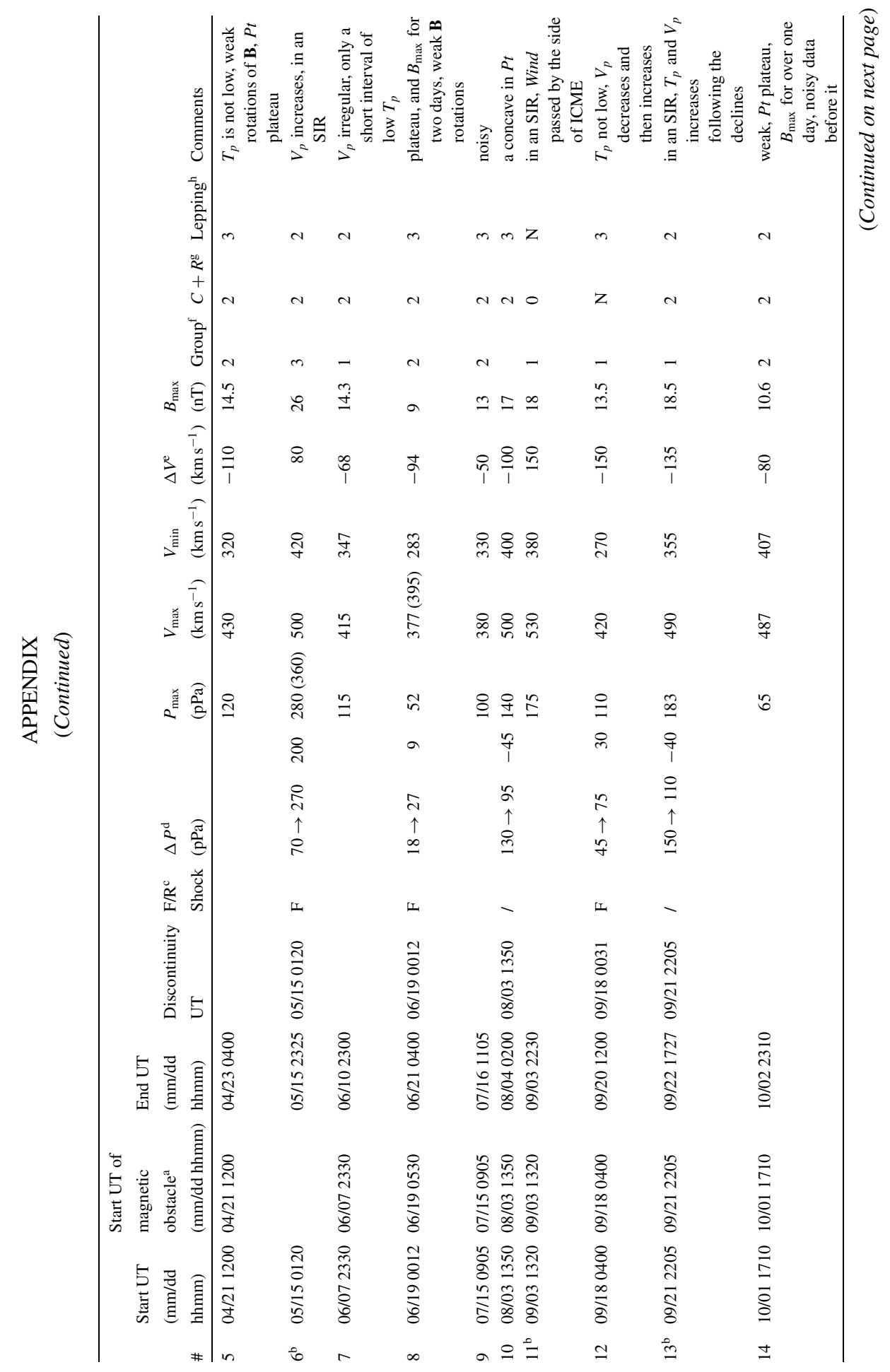




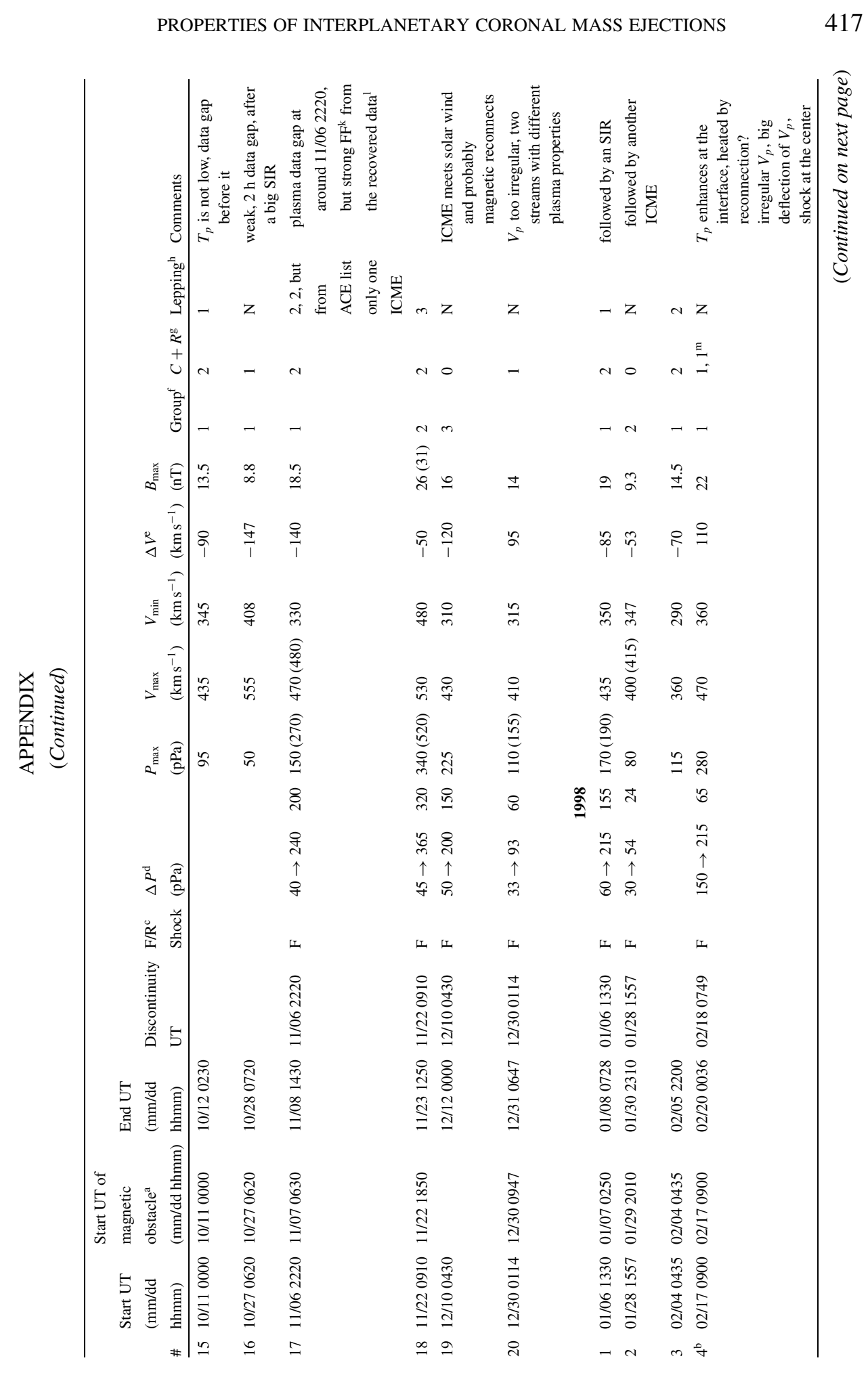




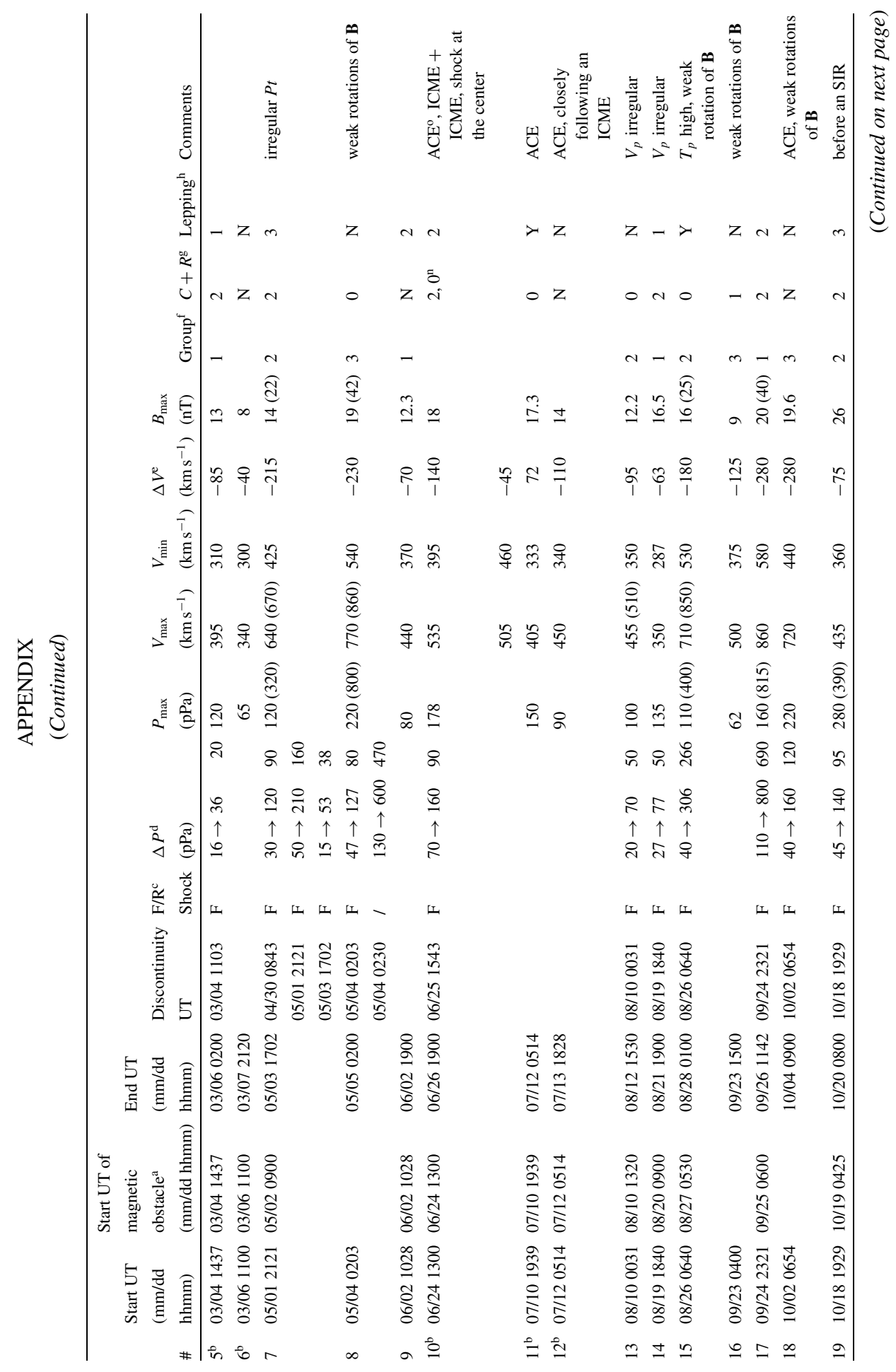


PROPERTIES OF INTERPLANETARY CORONAL MASS EJECTIONS

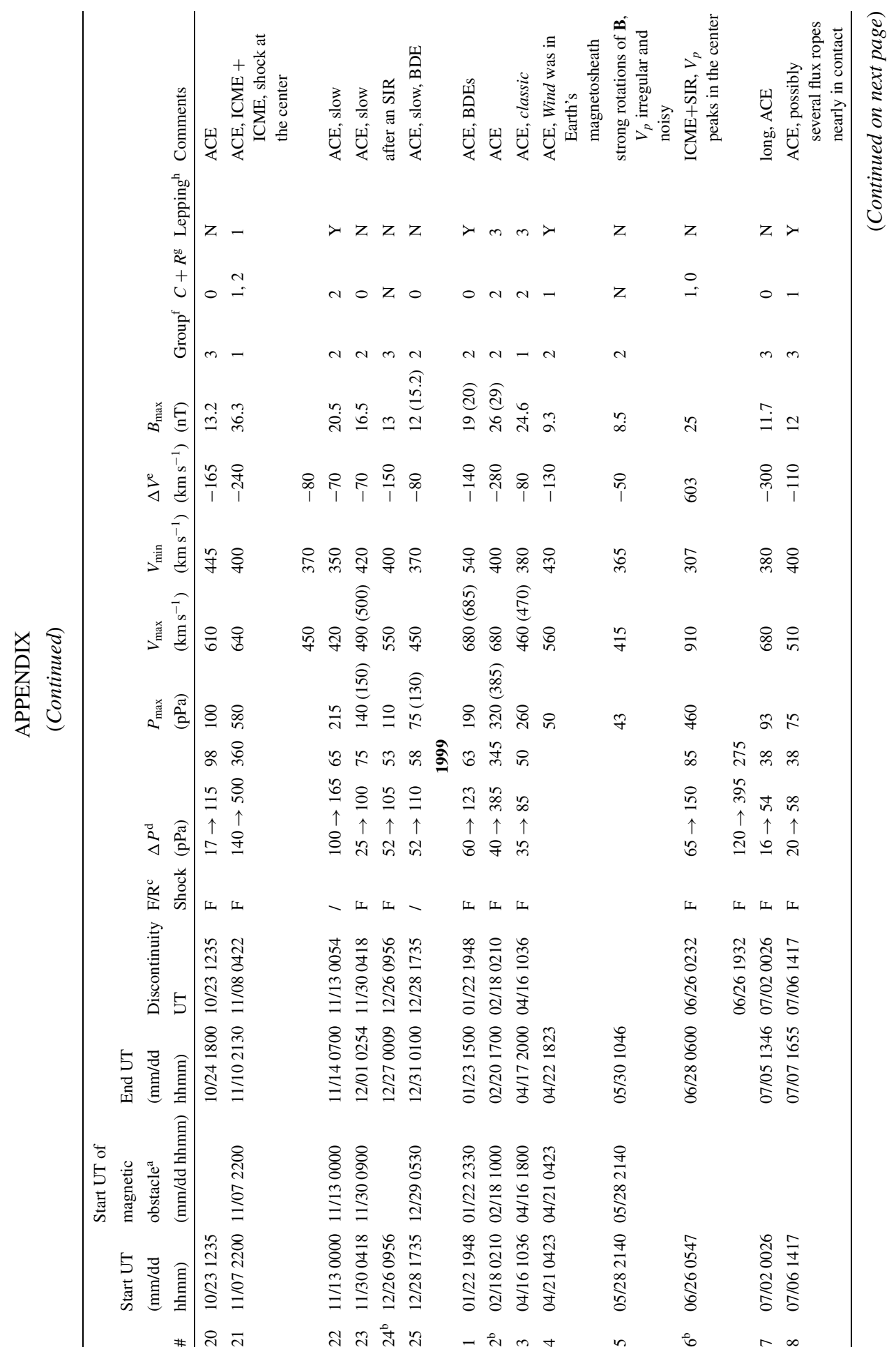




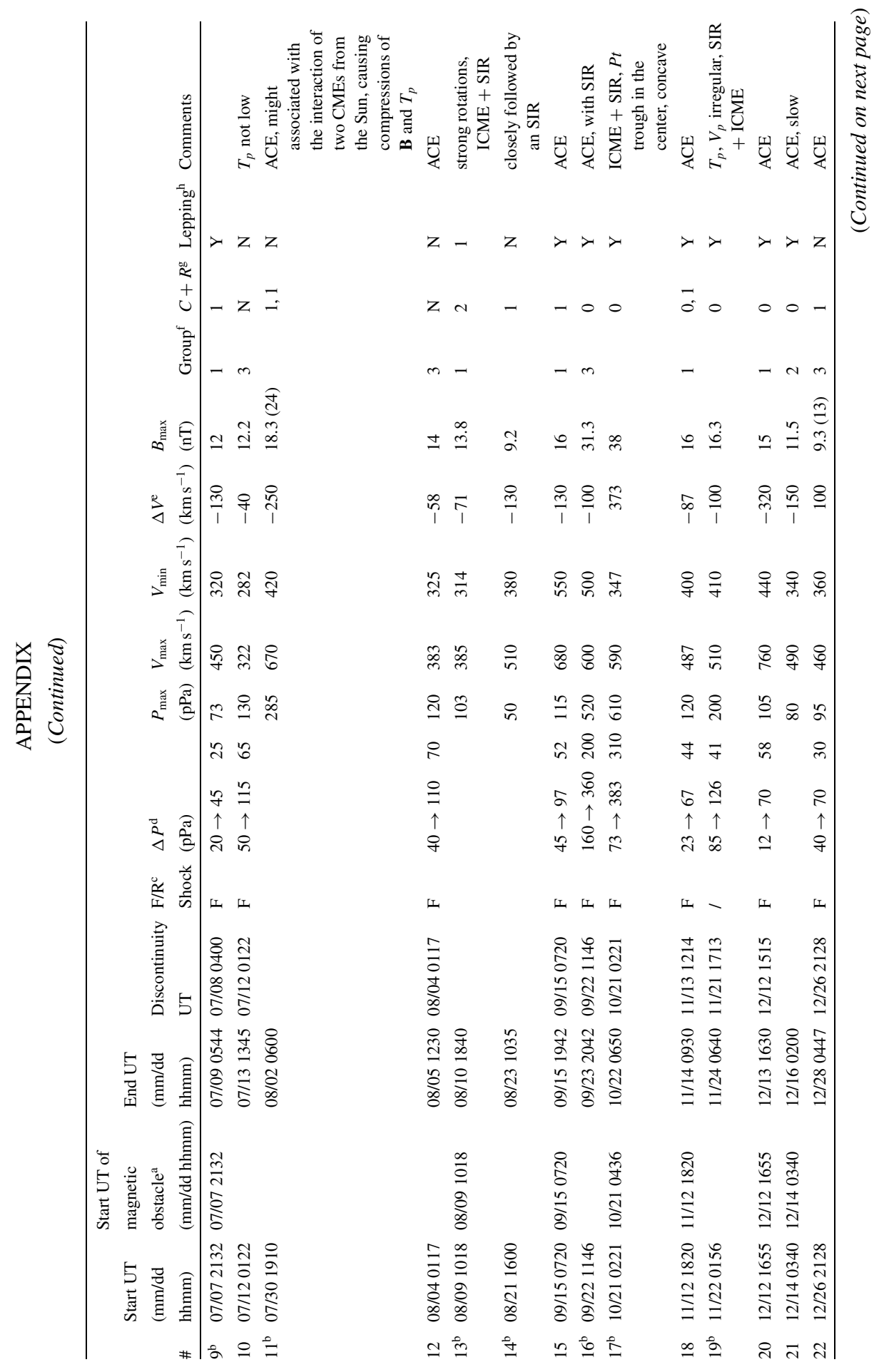




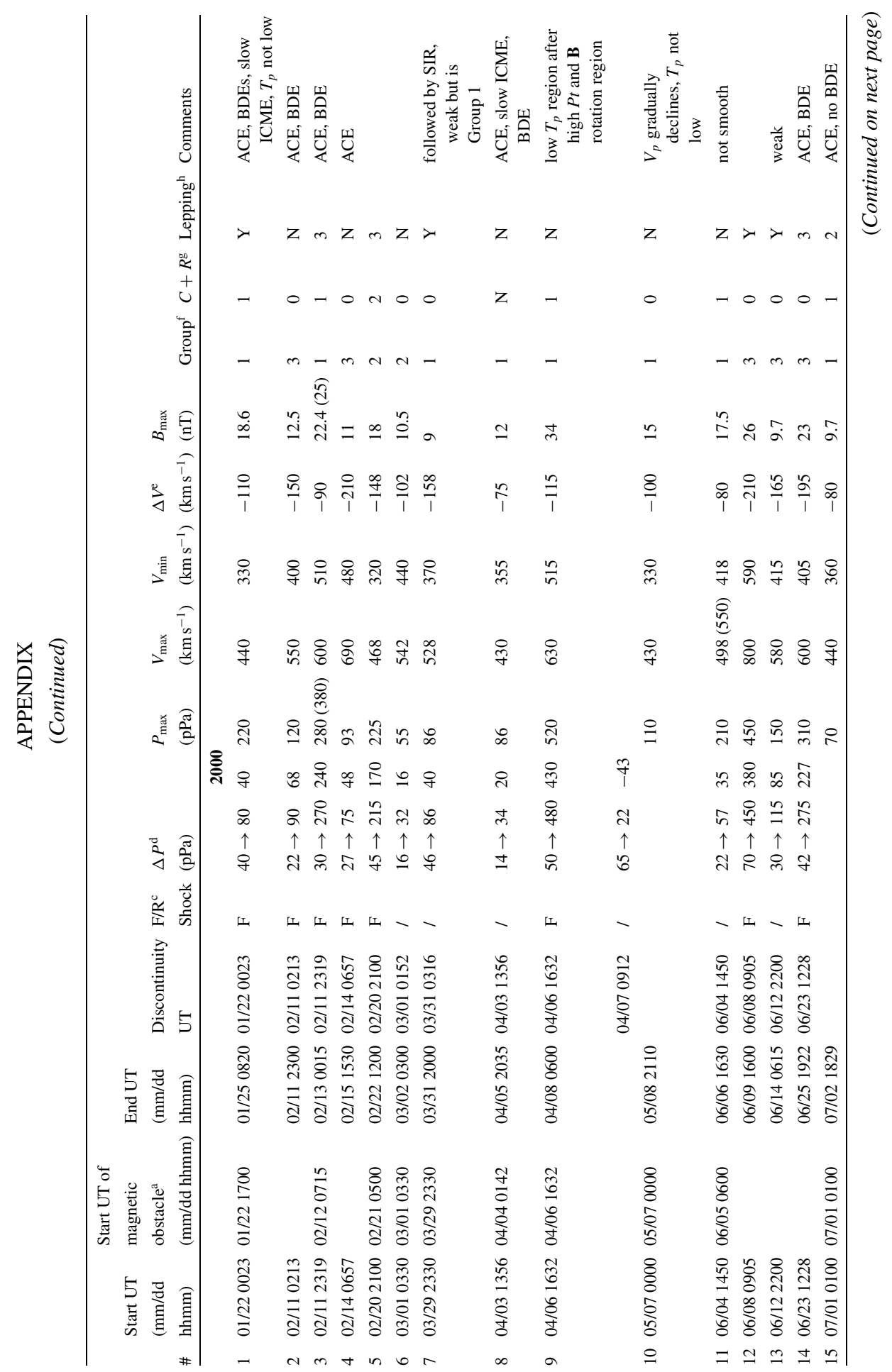




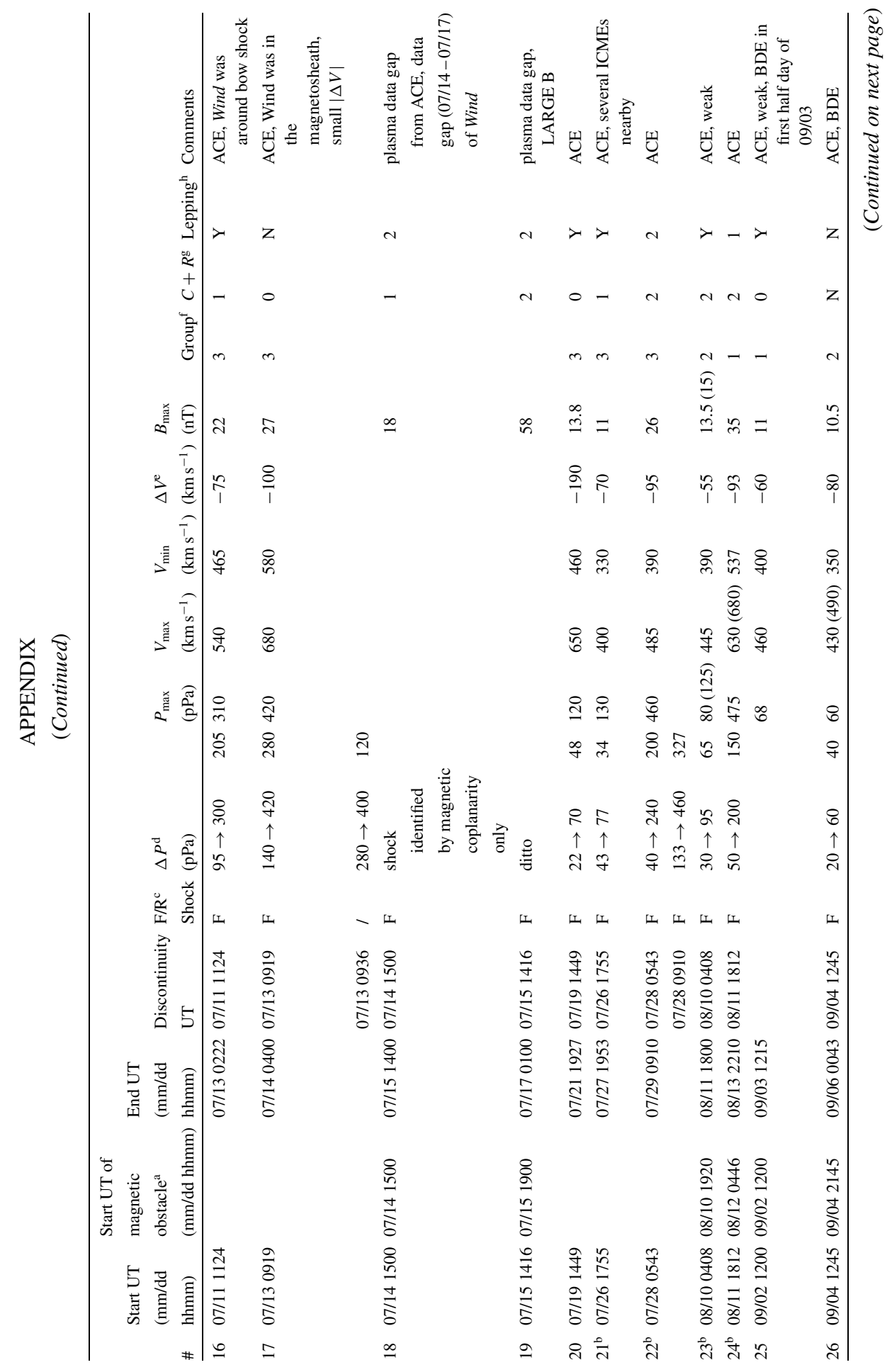


PROPERTIES OF INTERPLANETARY CORONAL MASS EJECTIONS

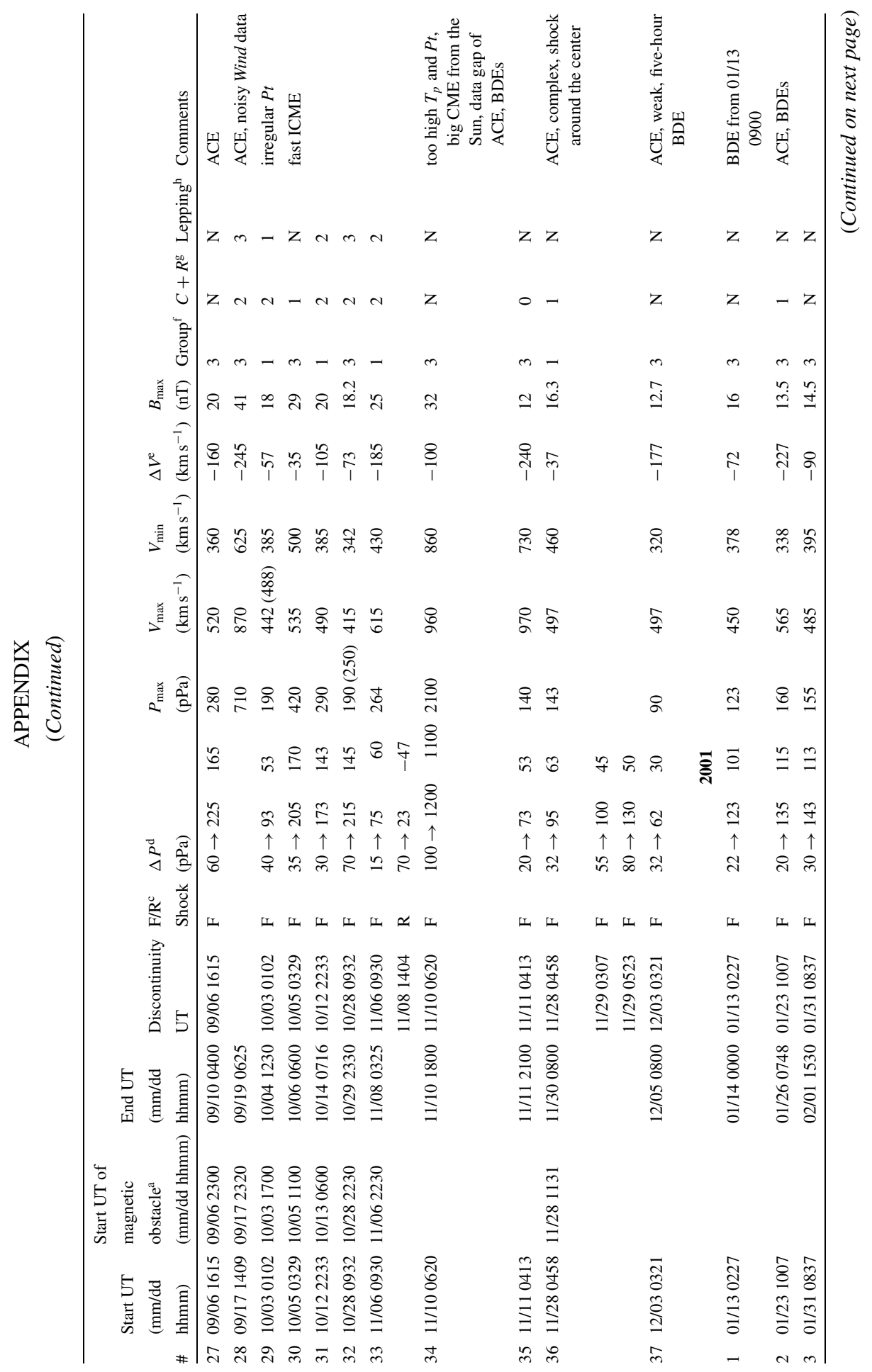


L. JIAN ET AL.

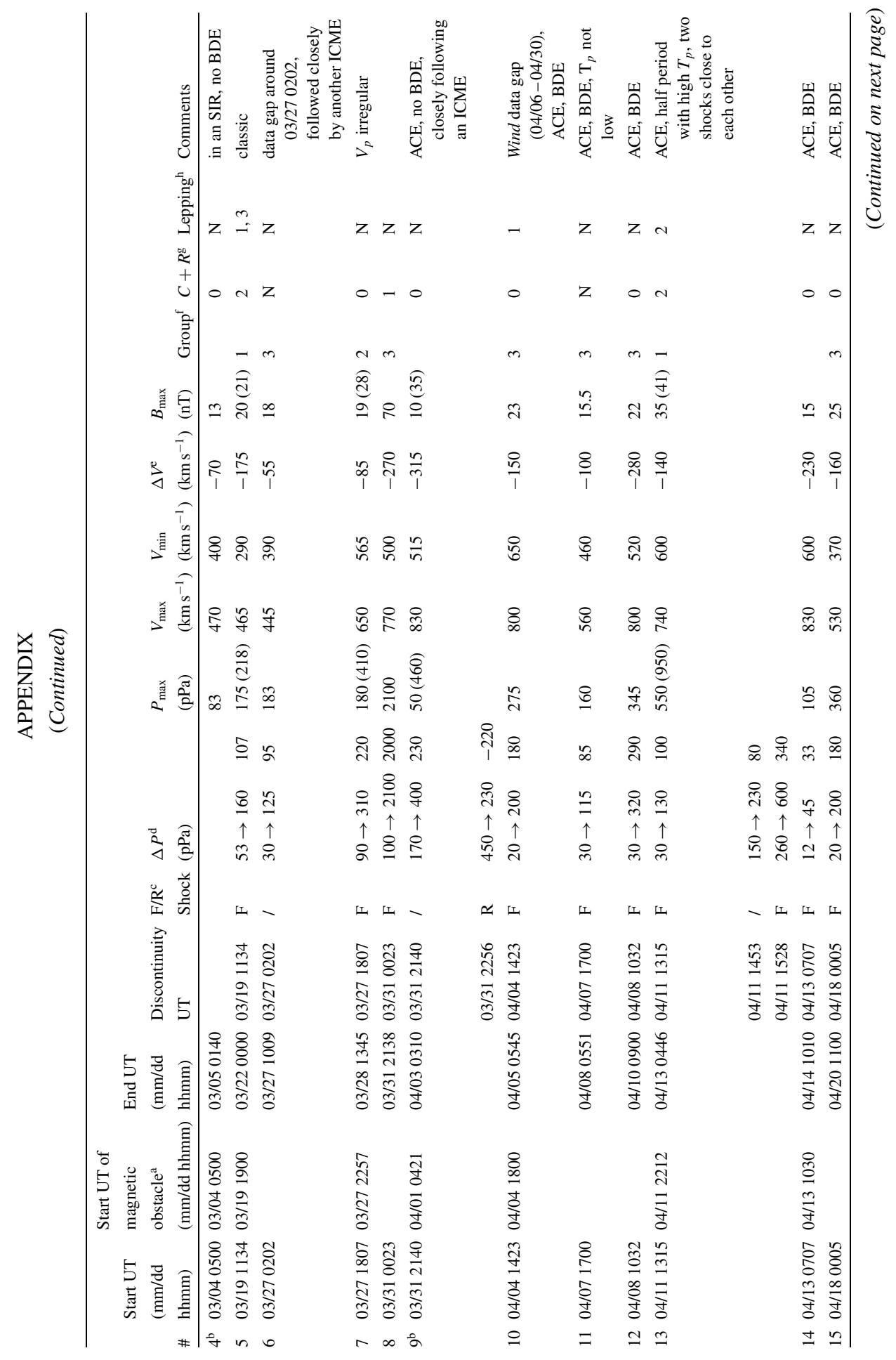


PROPERTIES OF INTERPLANETARY CORONAL MASS EJECTIONS

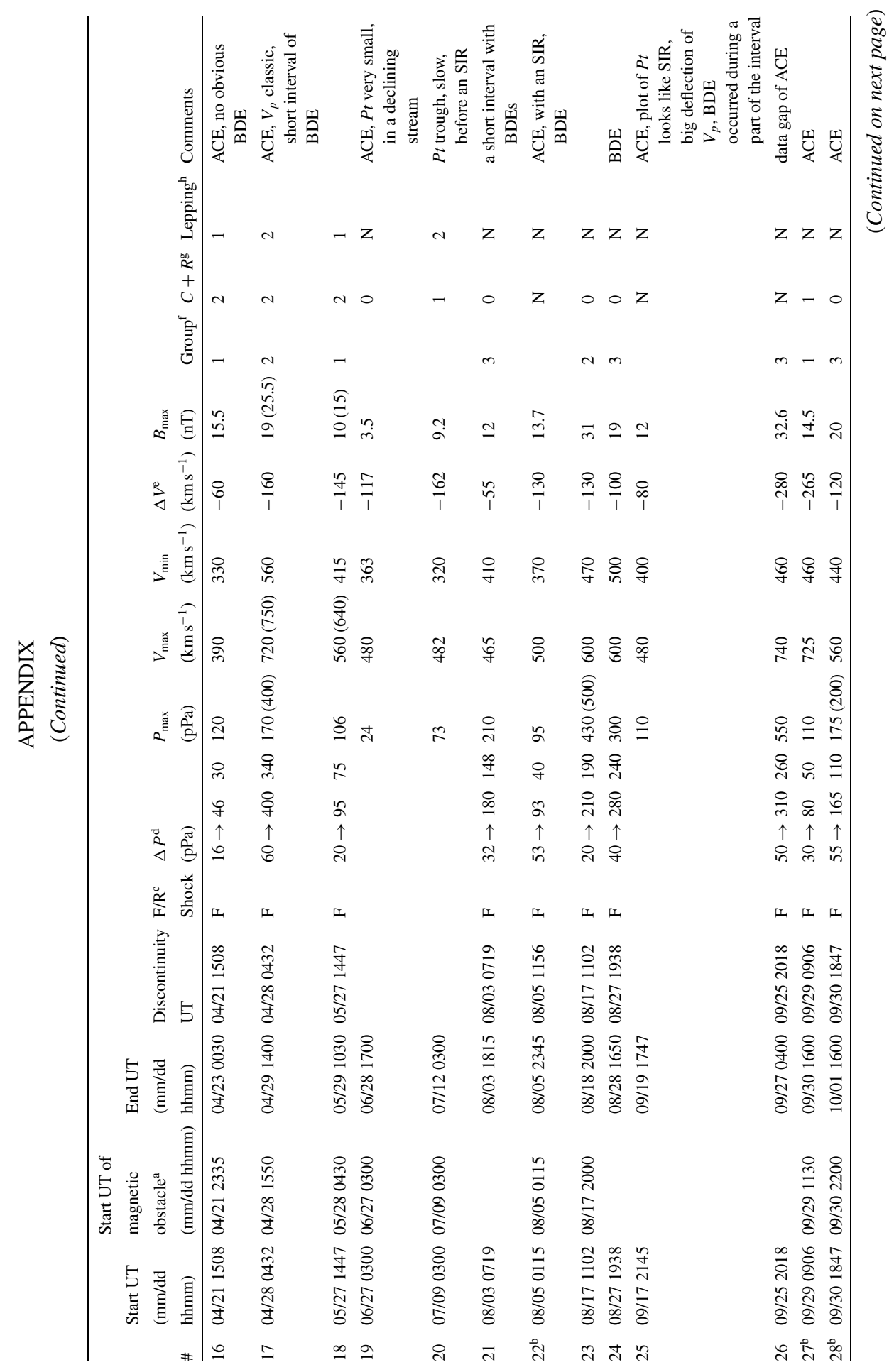




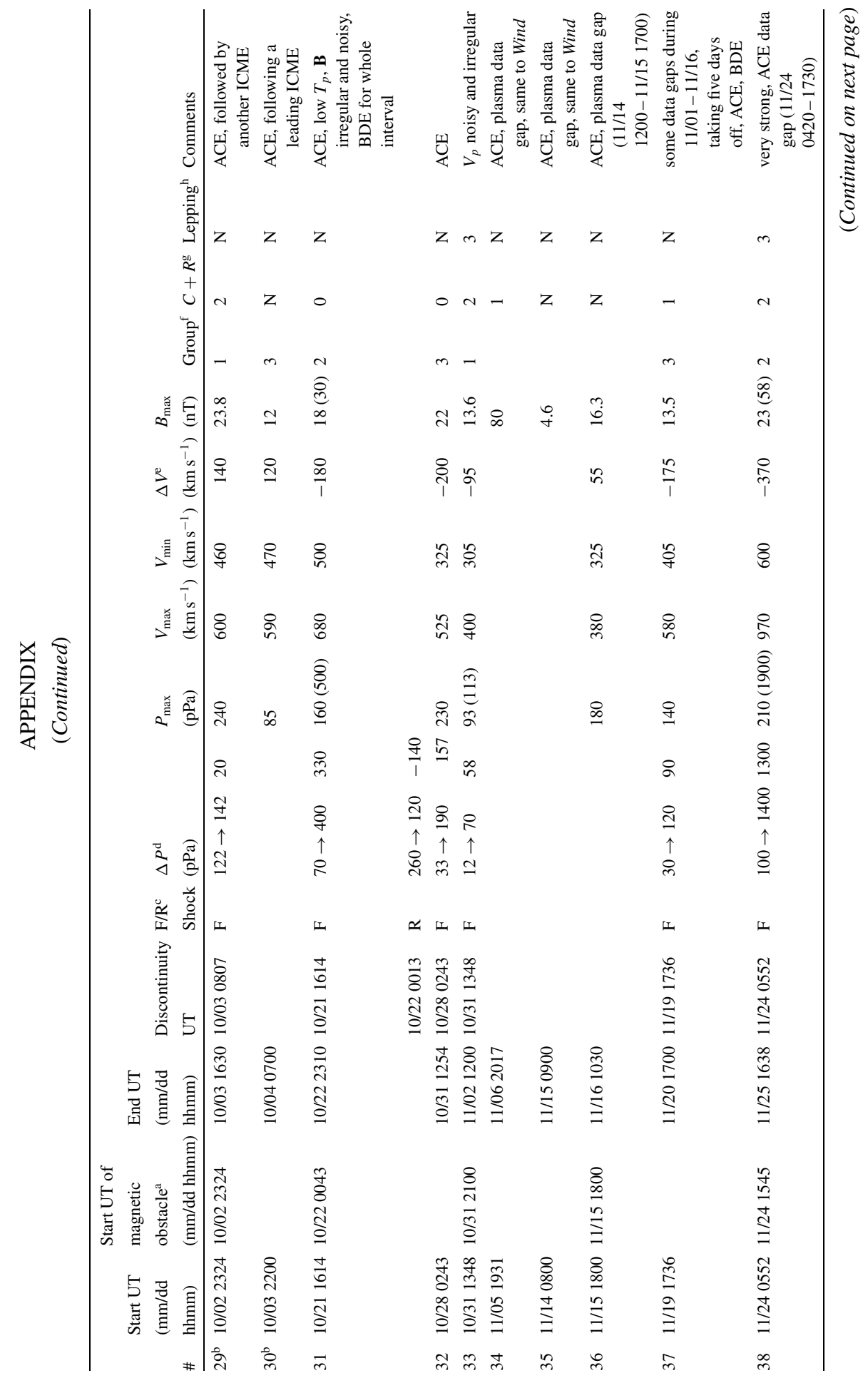




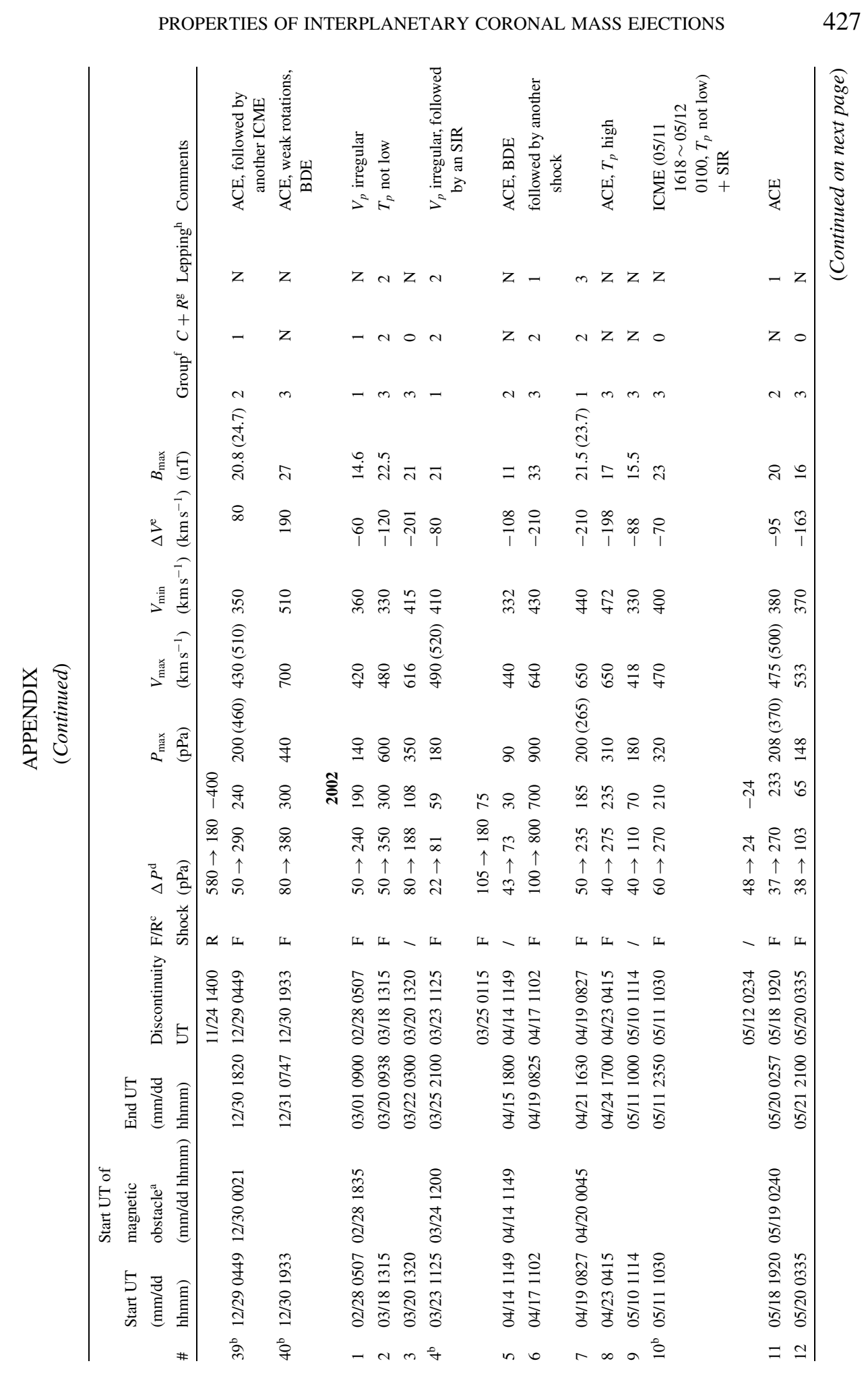




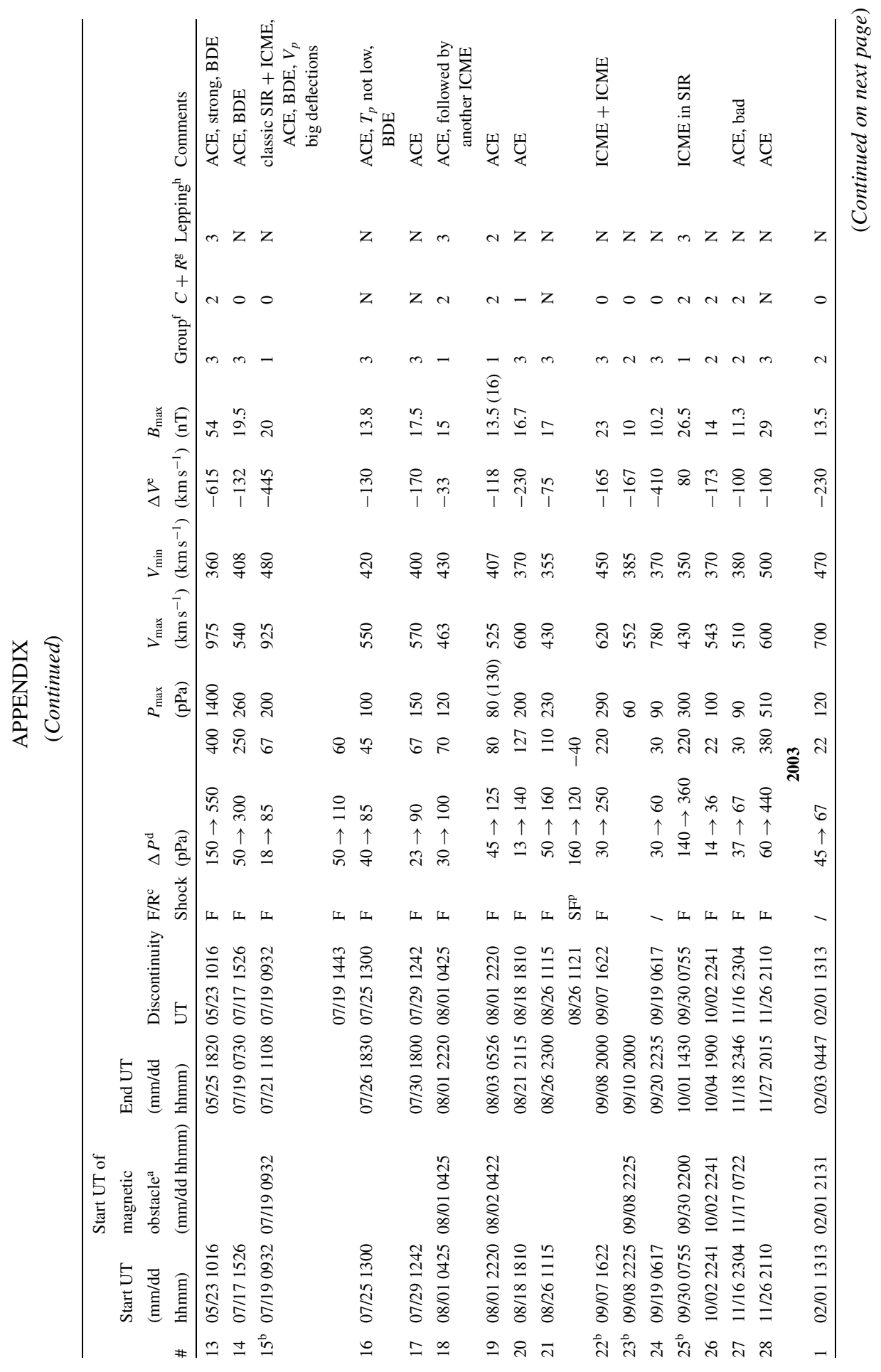




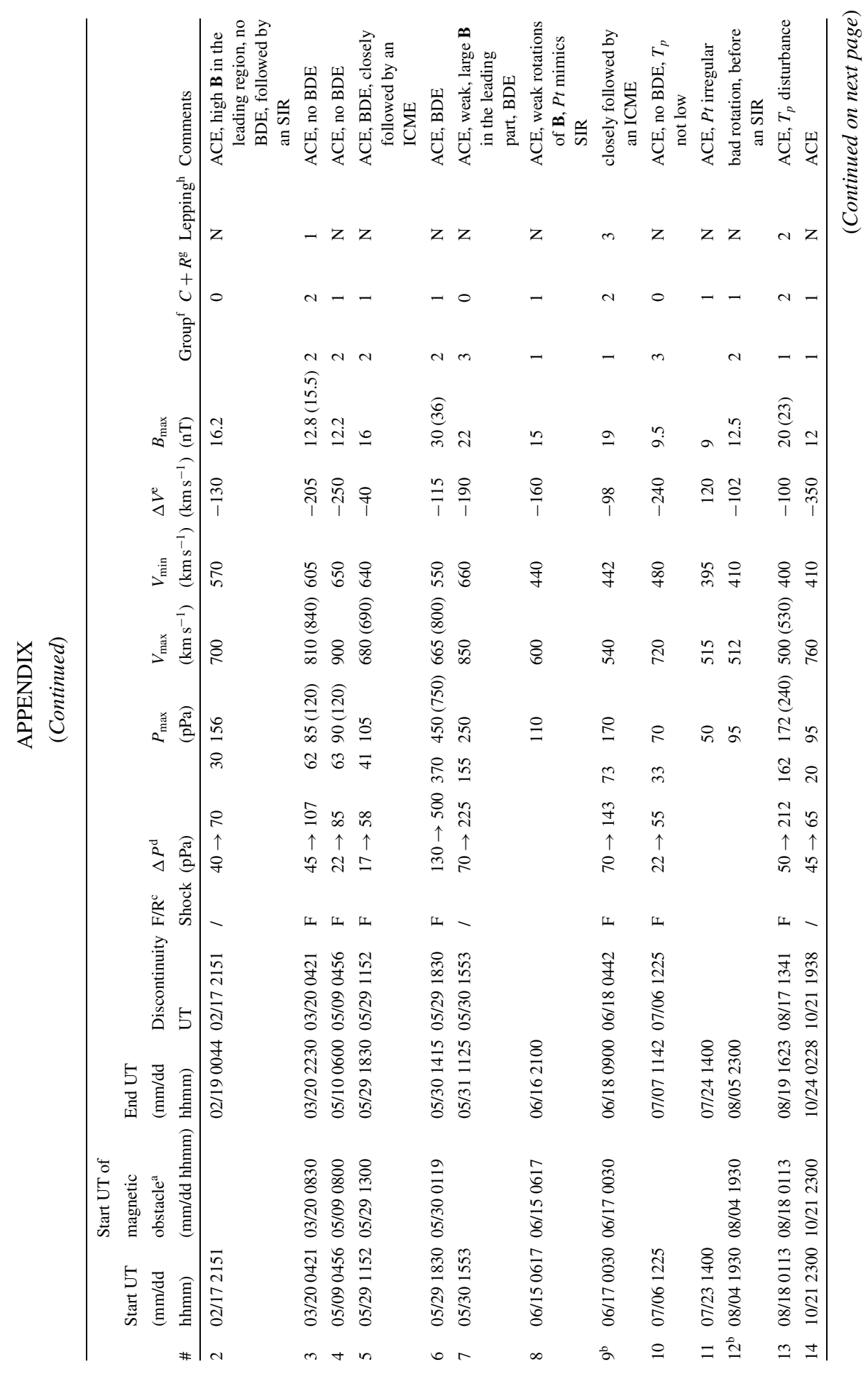




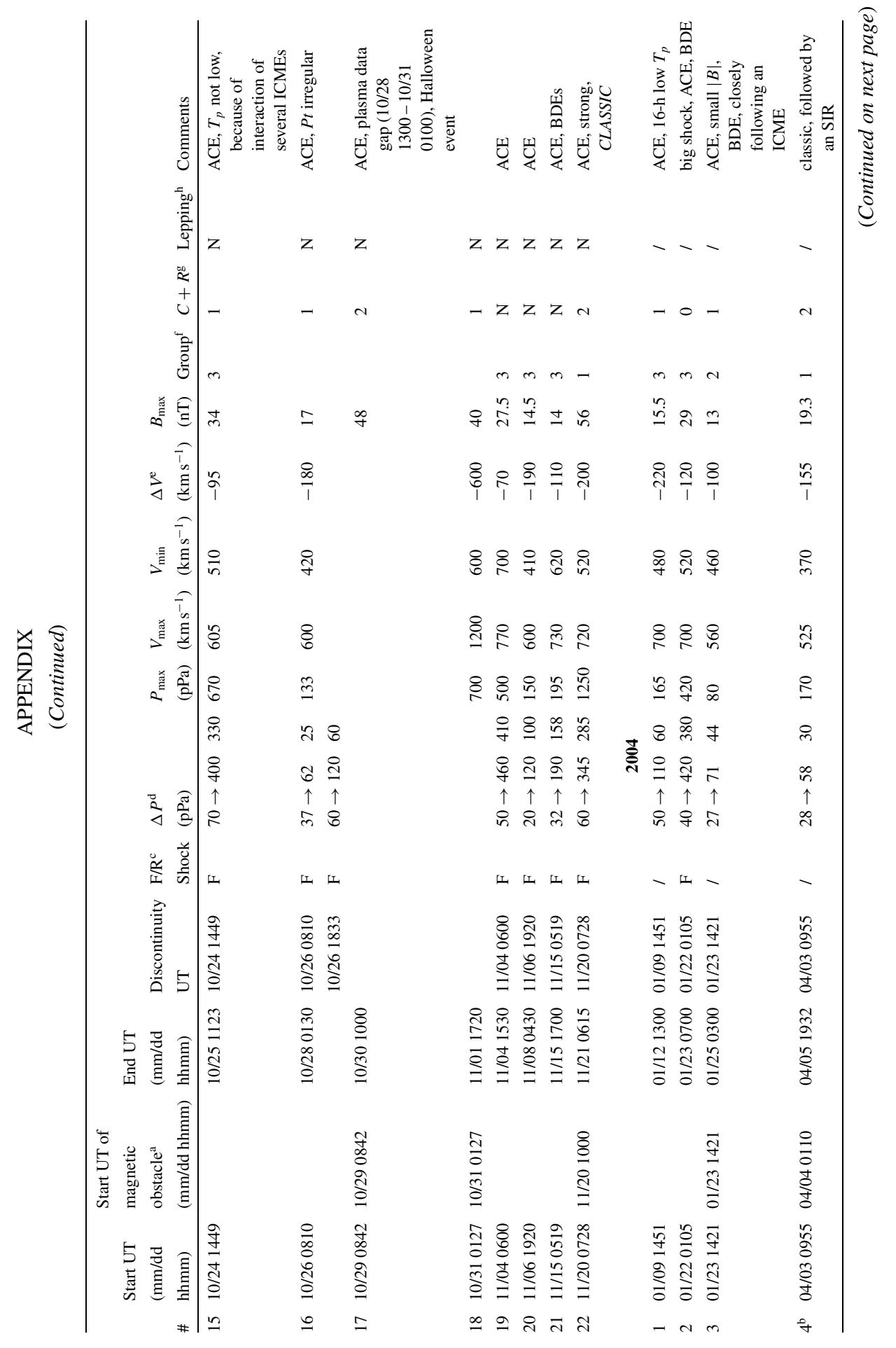




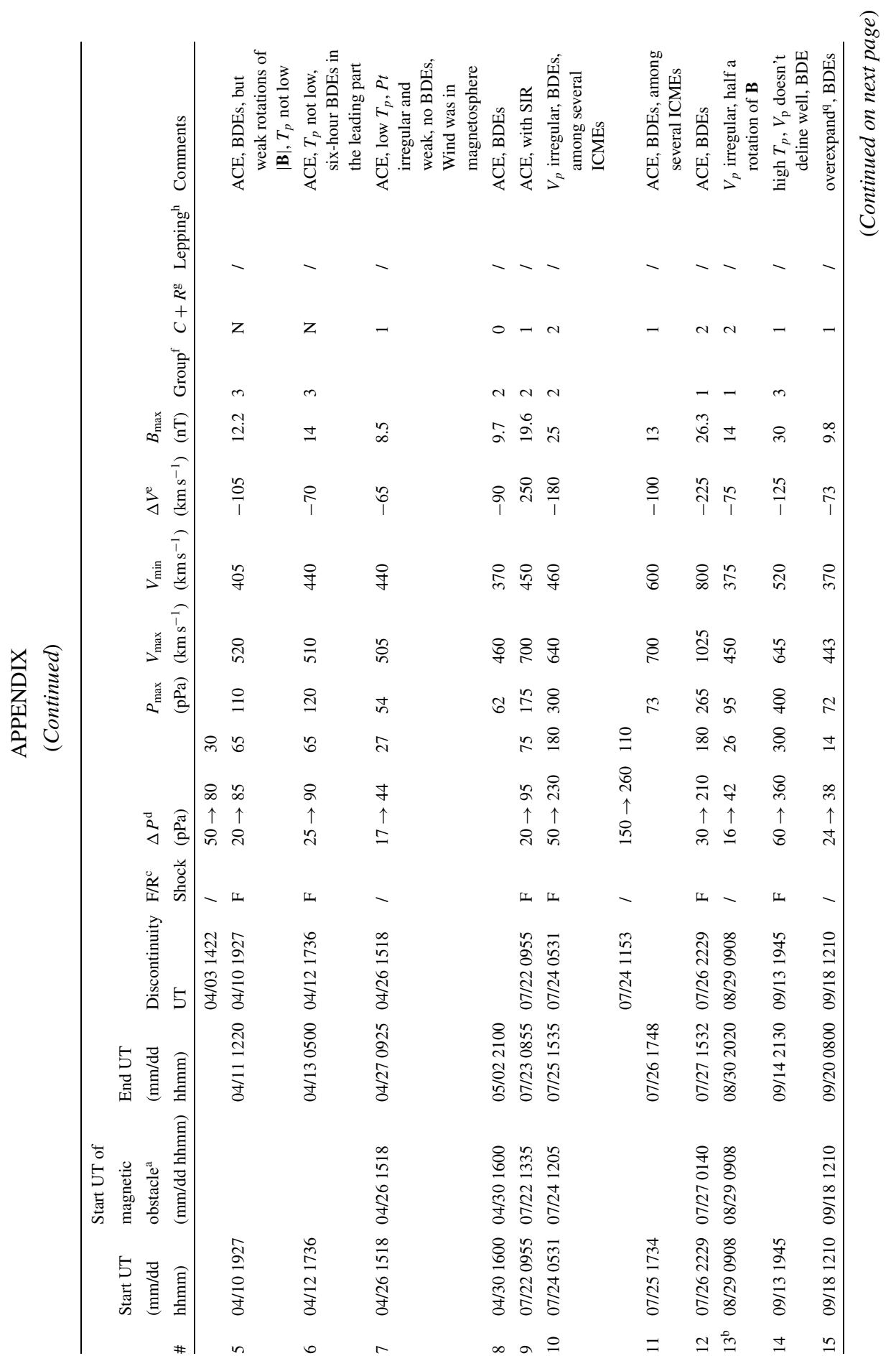




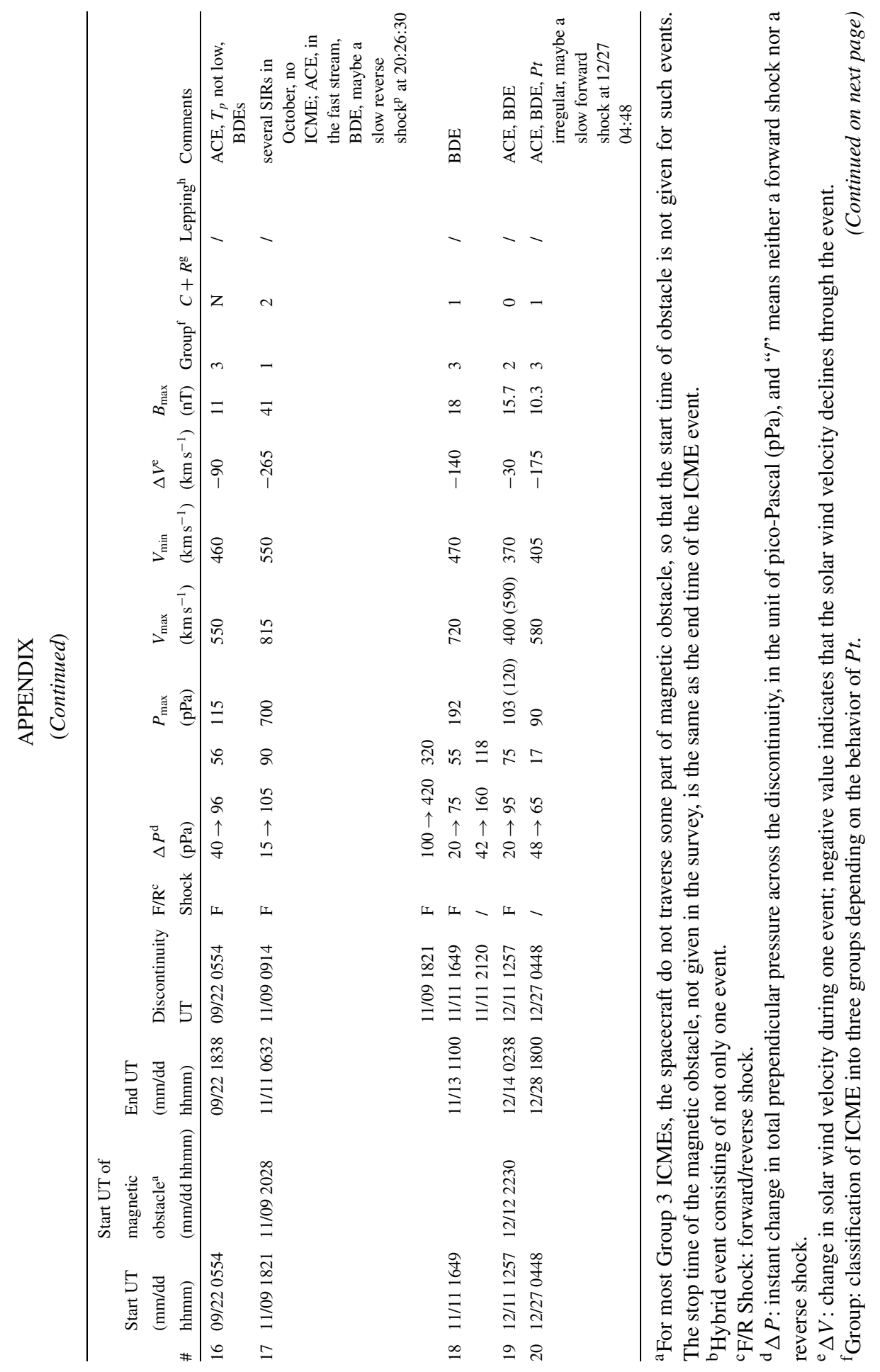




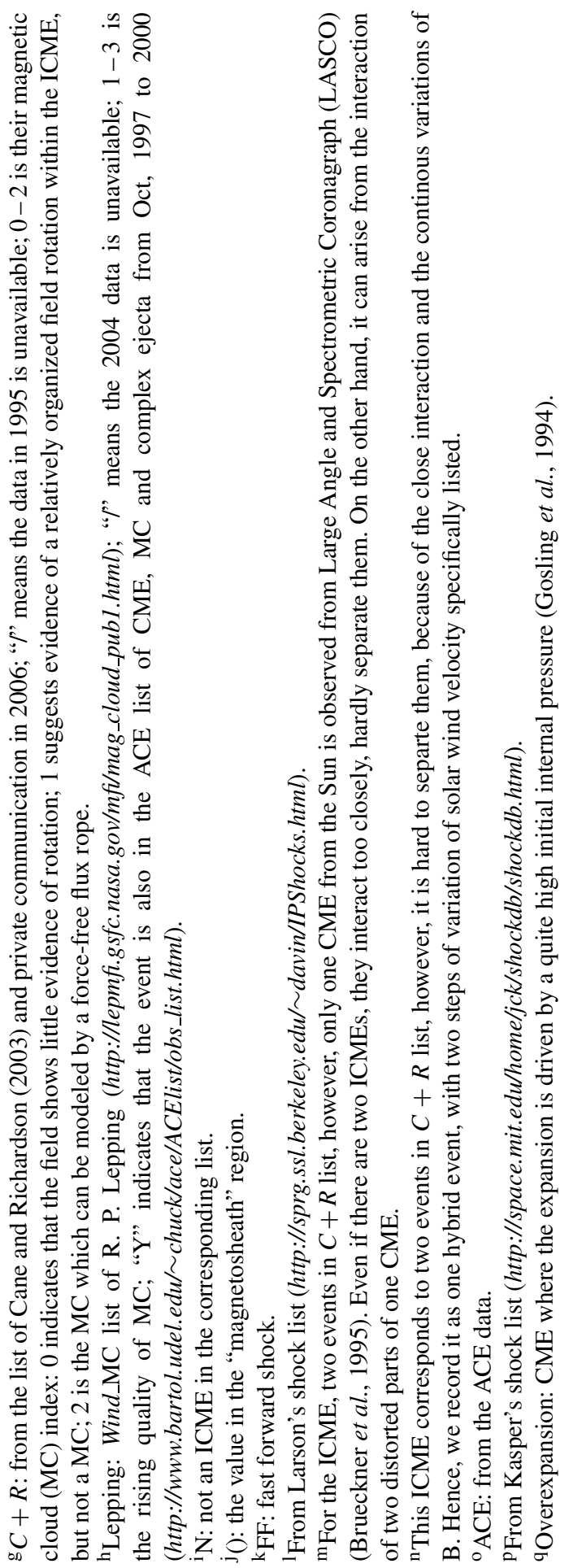




\section{References}

Bame, S.J., Asbridge, J.R., Feldman, W.C., Fenimore, E.E., and Gosling, J.T.: 1979, Solar Phys. 62, 179.

Brueckner, G.E., et al.: 1995, Solar Phys. 162, 357.

Burlaga, L.F.: 1991, in R. Schwenn and E. Marsch (eds.), Physics of the Inner Heliosphere II, SpringerVerlag, Berlin, p. 1.

Burlaga, L.F., Sittler, E., Mariani, F., and Schwenn, R.: 1981, J. Geophys. Res. 86, 6673.

Cane, H.V. and Richardson, I.G.: 2003, J. Geophys. Res. 108(A4), 1156, doi: 10.1029/2002JA009817. Cane, H.V., Richardson, I.G., and St. Cyr, O.C.: 2000, Geophys. Res. Lett. 27, 3591.

Feldman, W.C., Asbridge, J.R., Bame, S.J., and Montgomery, M.D.: 1973, J. Geophys. Res. 78(19), 3697.

Feldman, W.C., Anderson, R.C., Asbridge, J.R., Bame, S.J., Gosling, J.T., and Zwickl, R.D.: 1982, J. Geophys. Res. 87, 632.

Feldman, W.C., Anderson, R.C., Bame, S.J., Gosling, J.T., Zwickl, R.D., and Smith, E.J.: 1983, J. Geophys. Res. 88, 9949.

Goldstein, R., Neugebauer, M., and Clay, D.: 1998, J. Geophys. Res. 103, 4761.

Gosling, J.T.: 1990, in C.T. Russell, E.R. Priest, and L.C. Lee (eds.), Physics of Magnetic Flux Ropes, Geophys. Monogr. Ser., AGU, Washington, DC, 58, p. 343.

Gosling, J.T.: 1997, in N. Crooker, J.A. Joselyn, and J. Feynman (eds.), Coronal Mass Ejections, Geophys. Monogr. Ser., AGU, Washington, DC, 99, p. 9.

Gosling, J.T. and Pizzo, V.J.: 1999, Spa. Sci. Rev. 89, 21.

Gosling, J.T., Birn, J., and Hesse, M.: 1995, Geophys. Res. Lett. 22, 869.

Gosling, J.T., Pizzo, V., and Bame, S.J.: 1973, J. Geophys. Res. 78, 2001.

Gosling, J.T., Hildner, E., MacQueen, R.M., Munro, R.H., Poland, A.I., and Ross, C.L.: 1976, Solar Phys. 48, 389.

Gosling, J.T., Baker, D.N., Bame, S.J., Feldman, W.C., Zwickl, R.D., and Smith, E.J.: 1987, J. Geophys. Res. 92, 8519.

Gosling, J.T., Bame, S.J., Feldman, W.C., McComas, D.J., Phillips, J.L., and Goldstein, B.E.: 1993, Geophys. Res. Lett. 21, 2335.

Gosling, J.T., McComas, D.J., Phillips, J.L., Weiss, L.A., Pizzo, V.J., Goldstein, B.E., and Forsyth, R.J.: 1994, Geophys. Res. Lett. 21, 2271.

Gosling, J.T., McComas, D.J., Phillips, J.L., Pizzo, V.J., Goldstein, B.E., Forsyth, R.J., and Lepping, R.P.: 1995, Geophys. Res. Lett. 22, 1753.

Hirshberg, J. and Colburn, D.S.: 1969, Planet Spa. Sci. 17, 1183.

Hirshberg, J., Bame, S.J., and Robbins, D.E.: 1972, Solar Phys. 23, 467.

Hundhausen, A.J.: 1988, in V.J. Pizzo, T.E. Holzer, and D.G. Sime (eds.), Proceedings of the Sixth International Solar Wind Conference, Tech. Note NCAR/TN-306+Proc, 181, Natl. Cent. Atmos. Res., Boulder, CO.

Hundhausen, A.J.: 1993, J. Geophys. Res. 98, 13177.

Hundhausen, A.J.: 1999, in K.T. Strong, J.L.R. Saba, B.M. Haisch, and J.T. Schmelz (eds.), The Many Faces of the Sun: A Summary of the Results from NASA's Solar Maximum Mission, Springer, New York, p. 143.

Ipavich, F.M., Galvin, A.B., Gloeckler, G., Hovestadt, D., Bame, S.J., Klecker, B., Scholer, M., Fisk, L.A., and Fan, C.Y.: 1986, J. Geophys. Res. 91, 4133.

Issautier, K., Perche, C., Hoang, S., Lacombe, C., Maksimovic, M., Bougeret, J.-L., and Salem, C.: 2005, Advances in Spa. Res. 35, 2141.

Jian, L., Russell, C.T., Gosling, J.T., and Luhmann, J.G.: 2005a, AGU, Abstr. Spring, SH52A-11. 
Jian, L., Russell, C.T., Gosling, J.T., and Luhmann, J.G.: 2005b, in B. Fleck, T.H. Zurbuchen, and H. Lacoste (eds.), Proc. Solar Wind 11-SOHO 16, Connecting Sun and Heliosphere, ESA, Noordwijk, The Netherlands, p. 731.

Jian, L., Russell, C.T., Luhmann, J.G, and Skoug, R.M.: 2006, Solar Phys., in press.

Kahler, S.: 1988, in V.J. Pizzo, T.E. Holzer, and D.G. Sime (eds.), Proceedings of the Sixth International Solar Wind Conference, Tech. Note NCAR/TN-306+Proc, 181, Natl. Cent. Atmos. Res., Boulder, CO, p. 215.

Klein, L.W. and Burlaga, L.F.: 1982, J. Geophys. Res. 87, 613.

Lepping, R.P., Jones, J.A., and Burlaga, L.F.: 1990, J. Geophys. Res. 95, 11957.

Lepping, R. P., et al.: 1995, Spa. Sci. Rev. 71, 207.

Lepri, S.T., Zurbuchen, T.H., Fisk, L.A., Richardson, I.G., Cane, H.V., and Gloeckler, G.: 2001, J. Geophys. Res. 106, 29231.

Lin, R.P., et al.: 1995, Spa. Sci. Rev. 71, 125.

Lindsay, G.M., Russell, C.T., Luhmann, J.G., and Gazis, P.: 1994, J. Geophys. Res. 99(A1), 11.

Linker, J.A., Mikic, Z., Riley, P., Lionello, R., and Odstrcil, D.: 2003, in M. Velli, R. Bruno, and F. Malara (eds.), Proceedings of the 10th International Solar Wind Conference, AIP Conf. Proc. 679, Am. Inst. Phys., New York, p. 703.

Liu, Y., Richardson, J.D., and Belcher, J.W.: 2005, Plan. Spa. Sci. 53, 3.

Luhmann, J.G., Li, Y., Arge, C.N., Gazis, P.R., and Ulrich, R.: 2002, J. Geophys. Res. 107(A8), 1154, doi: 10.1029/2001JA007550.

Manchester, IV, W.B., Gombosi, T.I., Roussev, I., Ridley, A., De Zeeuw, D.L., Sokolov, I.V., Powell, K.G., and Tóth, G.: 2004, J. Geophys. Res. 109, A02107, doi: 10.1029/2003JA010150.

Marsden, R.G., Sanderson, T.R., Tranquille, C., Wenzel, K.-P., and Smith, E.J.: 1987, J. Geophys. Res. 92, 11009.

Marubashi, K: 1997, in N. Crooker, J.A. Joselyn, and J. Feynman (eds.), Coronal Mass Ejections, Geophys. Monogr. Ser., AGU, Washington, DC, 99, p. 147.

McComas, D.J., Bame, S.J., Barker, P., Feldman, W.C., Phillips, J.L., Riley, P., and Griffee, J.W.: 1998, Spa. Sci. Rev. 86, 563.

Montgomery, M.D., Asbridge, J.R., Bame, S.J., and Feldman, W.C.: 1974, J. Geophys. Res. 79, 3103.

Morrison, P.: 1956, Phys. Rev. 101, 1397.

Neugebauer, M. and Goldstein, R.: 1997, in N. Crooker, J.A. Joselyn, and J. Feynman (eds.), Coronal Mass Ejections, Geophys. Monogr. Ser., AGU, Washington, DC, 99, p. 245.

Newbury, J.A., Russell, C.T., Phillips, J.L., and Gary, S.P.: 1998, J. Geophys. Res. 103(A5), 9553.

Odstrcil, D. and Pizzo, V.J.: 1999a, J. Geophys. Res. 104, 483.

Odstrcil, D. and Pizzo, V.J.: 1999b, J. Geophys. Res. 104, 493.

Odstrcil, D. and Pizzo, V.J.: 1999c, J. Geophys. Res. 104, 28225.

Ogilvie, K.W., Scudder, J.D., and Sugiura, M.: 1971, J. Geophys. Res. 76, 8165.

Ogilvie, K.W., et al.: 1995, Spa. Sci. Rev. 71, 55.

Osherovich, V. and Burlaga, L.F.: 1997, in N. Crooker, J.A. Joselyn, and J. Feynman (eds.), Coronal Mass Ejections, Geophys. Monogr. Ser., AGU, Washington, DC, 99, p. 157.

Palmer, I.D., Allum, F.R., and Singer, S.: 1978, J. Geophys. Res. 83, 75.

Phillips, J.L., Bame, S.J., Feldman, W.C., Goldstein, B.E., Gosling, J.T., Hammond, C.M., McComas, D.J., Neugebauer, M., Scime, E.E., and Suess, S.T.: 1995, Science 268, 1030.

Richardson, I.G. and Cane, H.V.: 1993, J. Geophys. Res. 98, 15295.

Richardson, I.G. and Cane, H.V.: 1995, J. Geophys. Res. 100, 23397.

Richardson, I.G. and Cane, H.V.: 2004, Geophys. Res. Lett. 31, L18804, doi: 10.1029/2004GL020958.

Richardson, I.G. and Cane, H.V.: 2005, AGU, Abstr. Spring, SH43A-05

Richardson, I.G., Cane, H.V., and von Rosenvinge, T.T.: 1991, J. Geophys. Res. 96, 7853.

Riley, P., Linker, J.A., Mikić, Z., Odstrcil, D., Zurbuchen, T.H., Lario, D., and Lepping, R.P.: 2003, J. Geophys. Res. 108(A7), 1272. 
Riley, P., Schatzman, C., Cane, H.V., Richardson, I.G., and Gopalswamy, N.: 2006, Astrophys. J. 647, 648.

Russell, C.T. and McPherron, R.L.: 1973, Spa. Sci. Rev. 15, 205.

Russell, C.T. and Shinde, A.A.: 2003, Solar Phys. 216, 285.

Russell, C.T. and Shinde, A.A.: 2005, Solar Phys. 229, 323.

Russell, C.T., Shinde, A.A., and Jian, L.: 2005, Adv. Space Res. 35, 2178.

Rust, D.M.: 1999, Geophys. Monogr. 109, 213.

Shodhan, S., Crooker, N.U., Kahler, S.W., Fitzenreiter, R.J., Larson, D.E., Lepping, R.P., Siscoe, G.L., and Gosling, J.T.: 2000, J. Geophys. Res. 105(A12), 27261.

Smith, C.W., L'Heureux, J., Ness, N.F., Acuña, M.H., Burlaga, L.F., and Scheifele, J.: 1998, Spa. Sci. Rev. 86, 613.

Spreiter, J.R., Summers, A.L., and Alksne, A.Y.: 1966, Plan. Spa. Sci. 14, 223.

Steinberg, J.T., Gosling, J.T., Skoug, R.M., and Wiens, R.C.: 2005, J. Geophys. Res. 110, A06103, doi: 10.1029/2005JA011027.

Zwickl, R.D., Asbridge, J.R., Bame, S.J., Feldman, W.C., Gosling, J.T., and Smith, E.J.: 1983, in M. Neugebauer (ed.), Solar Wind Five, NASA Conf. Publ. 2280, NASA, Washington, DC, p. 711. 